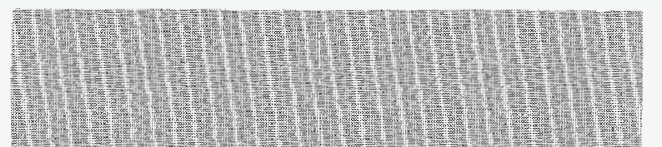

OAK RIDGE

NATIONAL

LABORATORY
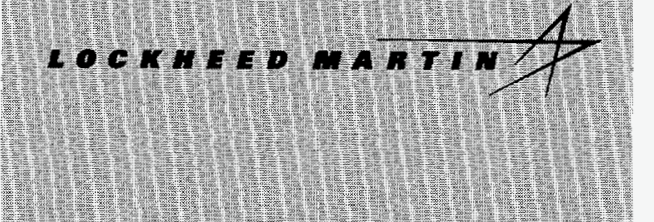

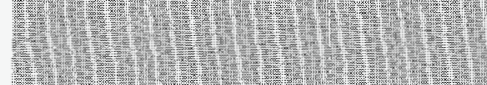

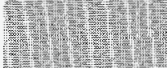

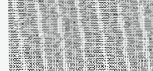

औint:

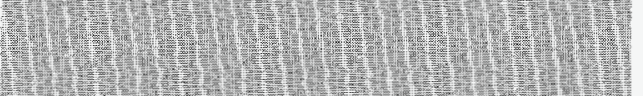

(4)

(5)

1)

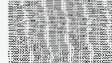

(1)

(4)

(4)

MANAGED AND OPERATED BY

LOCKHEED MARTIN ENERGY RESEARCH CORPORATION

FOR THEUUMTEO STATES

DEPARTMENT OF ENERGY

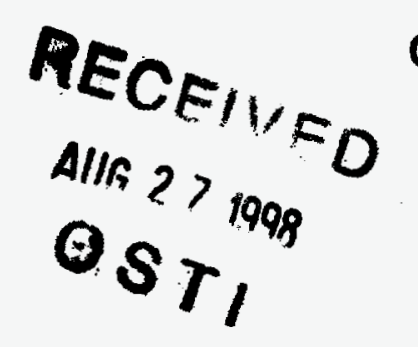

ORNL/M-6576

N. M. Larson

\title{
Introduction to the Theory and Analysis of Resolved (and Unresolved) Neutron Resonances via SAMMY
}

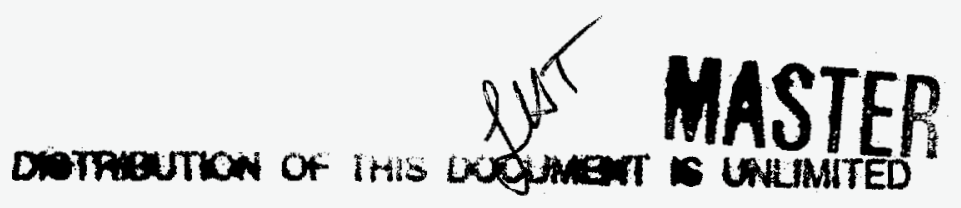


This report hes been reproduced directly from the best available copy.

Available to DOE and DOE contractors from the Office of Scientific and Technical information, P.O. Box 62, Oak Ridge, TN 37831; prices available from (615) 576-8401, FTS 626-8401.

Available to the public from the National Technical information Service, U.S. Department of Commerce, 5285 Port Royal Rd., Springfield, VA 22161.

This report was prepared as an account of work sponsored by an agency of the United States Government. Neither the United States Government nor any agency thereof, nor any of their empioyees, makes any warranty, express or implied, or assumes any legal liability or responsibility for the accuracy, com pleteness, or usefulness of any information, apparatus, product, or process disclosed, or represents that its use would not infringe privately owned rights. Reference herein to any specific commercial product, process, or service by trade name, trademark, manufecturer, or otherwise, does not necessarily constitute or imply its endorsement, recommendation. or favoring by the United States Government or any agency thereof. The views and opinions of authors expressed herein do not necessarily state or reflect those of the United States Government or any agency thereot. 


\section{DISCLAIMER}

Portions of this document may be illegible in electronic image products. Images are produced from the best available original document. 
Computational Physics and Engineering Division

\title{
INTRODUCTION TO THE THEORY AND ANALYSIS OF RESOLVED
} (AND UNRESOLVED) NEUTRON RESONANCES VIA SAMMY

N. M. Larson

July 1998

\author{
Prepared by the \\ Oak Ridge National Laboratory \\ Oak Ridge, Tennessee 37831-6370 \\ Managed by \\ LOCKHEED MARTIN ENERGY RESEARCH \\ for the \\ U.S. DEPARTMENT OF ENERGY \\ under contract DE-AC05-96OR22464
}





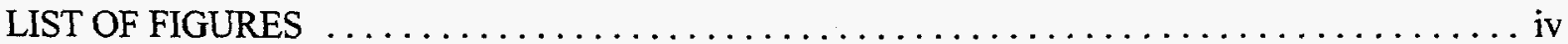

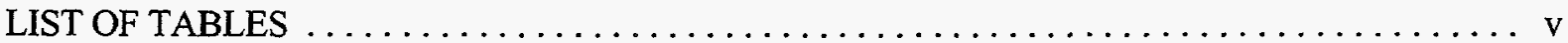

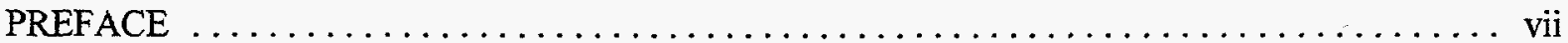

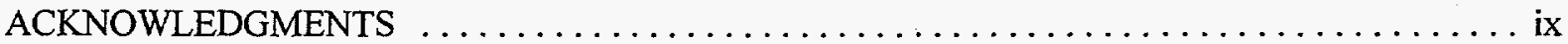

1 Overview $\ldots \ldots \ldots \ldots \ldots \ldots \ldots \ldots \ldots \ldots \ldots \ldots \ldots \ldots \ldots \ldots \ldots \ldots \ldots \ldots \ldots \ldots$

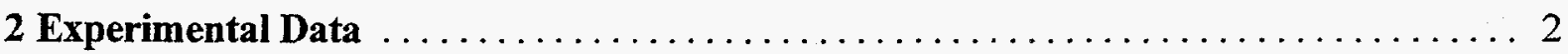

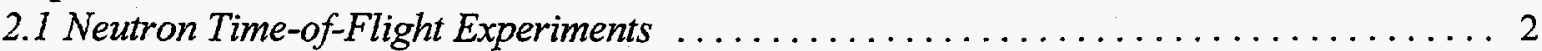

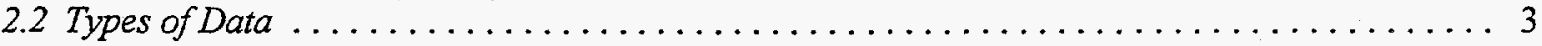

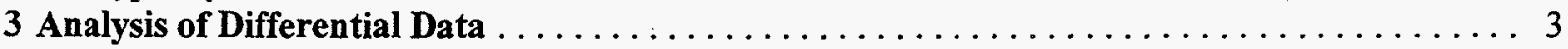

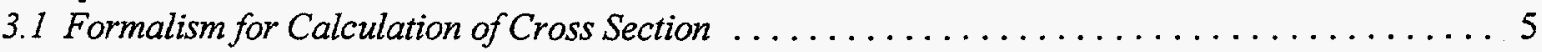

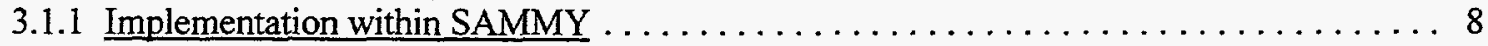

3.2 Mathematical Description of Experimental Effects $\ldots \ldots \ldots \ldots \ldots \ldots \ldots \ldots \ldots \ldots$

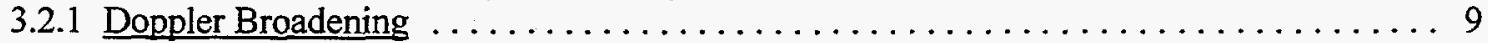

3.2.1.1 Derivation of Free Gas Model . . . . . . . . . . . . . . . . . . . 10

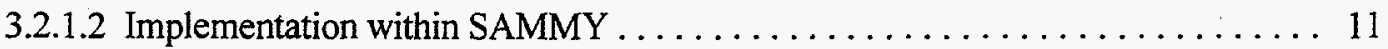

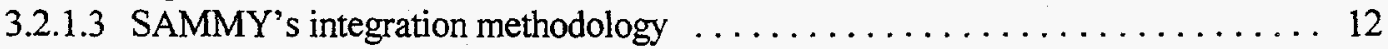

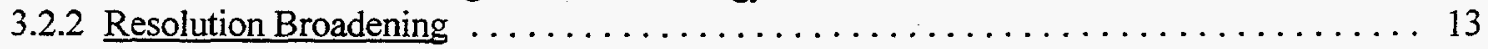

3.2.2.1 Gaussian and/or Exponential Resolution-Broadening Function . . . . . . . . 13

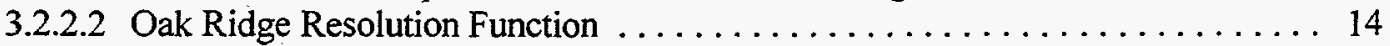

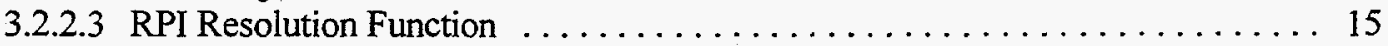

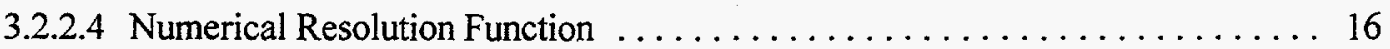

3.2 .3 Explicit Normalization and/or Background Functions $\ldots \ldots \ldots \ldots \ldots \ldots \ldots \ldots \ldots$

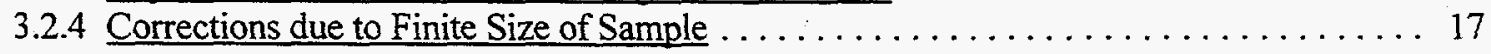

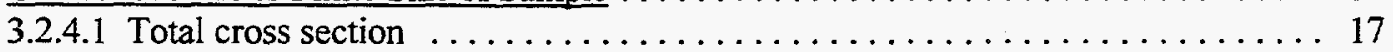

3.2.4.2 Partial cross sections . . . . . . . . . . . . . . . . . . . . 17

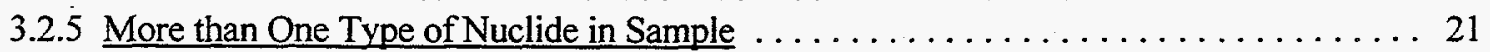

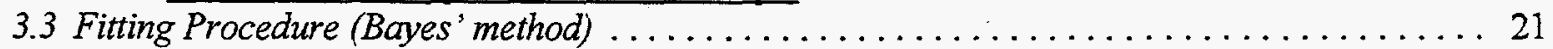

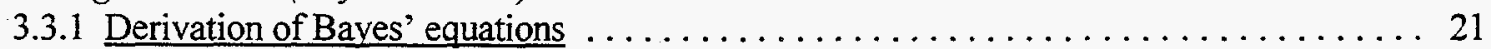

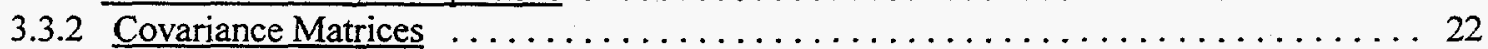

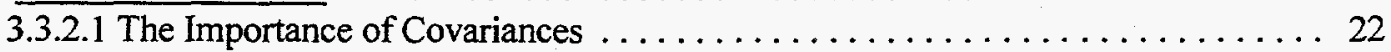

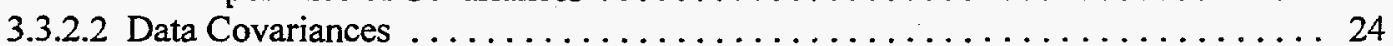

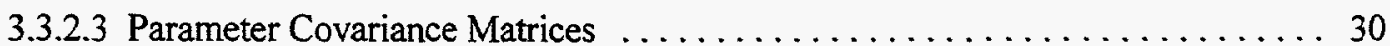

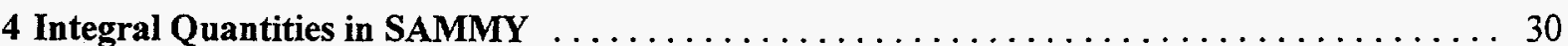

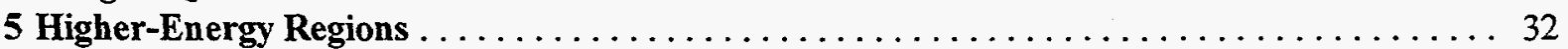

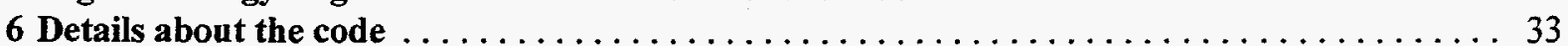

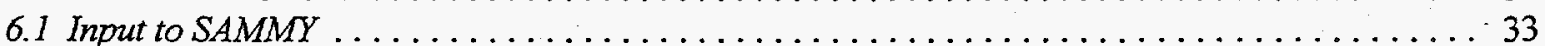

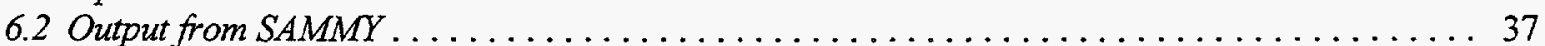

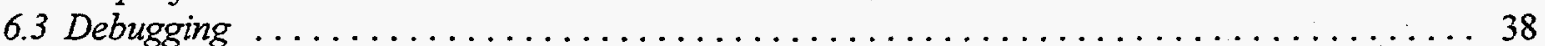

6.4 SAMMY features not mentioned elsewhere $\ldots \ldots \ldots \ldots \ldots \ldots \ldots \ldots \ldots \ldots \ldots \ldots \ldots$

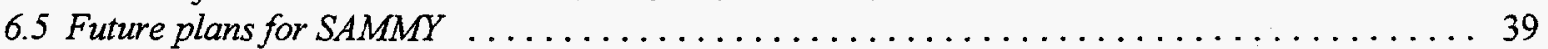

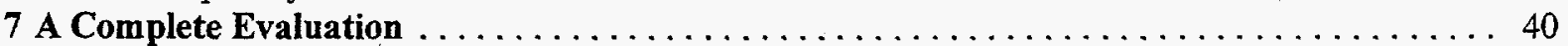

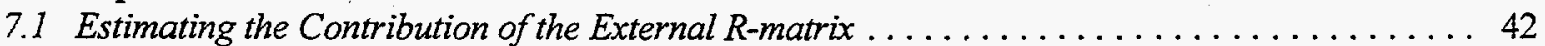

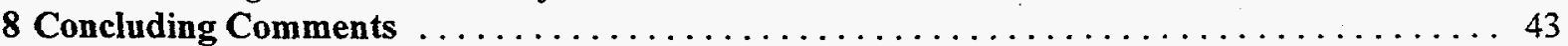

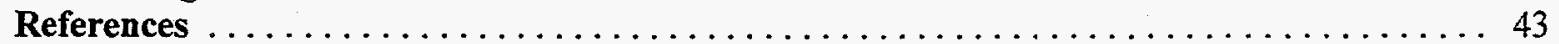


1 Schematic of a neutron time-of-flight transmission experiment.

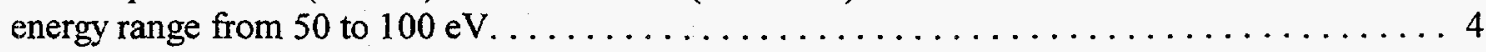

$3 \quad{ }^{235} \mathrm{U}$ experimental (crosses) and calculated (solid curve) total cross section, fission cross section, and capture cross section in the energy range from 400 to $500 \mathrm{eV} . \ldots \ldots \ldots \ldots$

4 Schematic of entrance and exit channels as used in scattering theory $\ldots \ldots \ldots \ldots \ldots \ldots$

5 Capture cross section for one resonance in ${ }^{58} \mathrm{Ni}$, without Doppler broadening (dashed curve) and with Doppler broadening at $300 \mathrm{~K}$ via the free-gas model (solid curve) $\ldots \ldots \ldots \ldots \ldots$

6 Idealized schematic of a capture experiment using (a) a very thin sample, (b) a slightly thicker sample, and (c) a realistically thick sample. . . . . . . . . . . . . 18

7 Geometry for the single-scattering correction to capture or fission yield, for a neutron

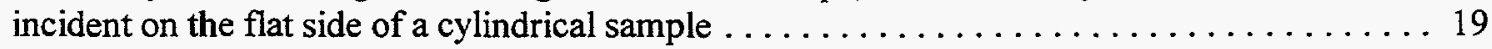

8 The $1.15-\mathrm{keV}^{56} \mathrm{Fe}$ resonance in natural iron capture data of $\mathrm{R}$. Spencer et al. . . . . . 20

9 Artificial, uranium-like fission "experimental data" (crosses) and SAMMY fit (solid curve), to illustrate the use of covariance matrices in calculating group cross-section uncertainties. . . . 24

10 The fit to the "fission cross section for ${ }^{241}$ Am," using Method 1 (fit reduced data using

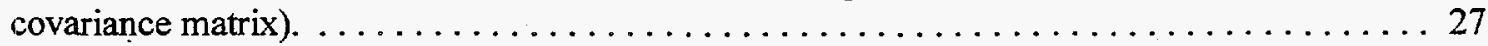

11 The same fit shown in Fig. 10, with the dotted curve representing the SAMMY fit (as shown in solid curve in Fig. 10), and the solid curve showing the SAMMY fit adjusted by the "true" values of the data-reduction parameters $(1.17 \times$ dotted +4.91$) \ldots \ldots \ldots \ldots \ldots \ldots \ldots \ldots \ldots$

12 Fission cross section for ${ }^{241} \mathrm{Am}$ using Method 2, "a better approach" (fit raw data using data-

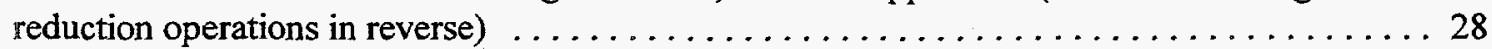

13 Fission cross section for ${ }^{241}$ Am using Method 3, "the usual approach" (fit reduced data using

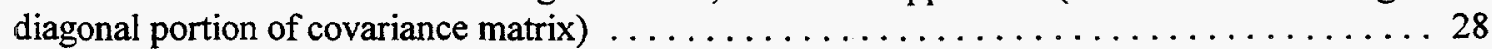

14 Fission cross section for ${ }^{241}$ Am using Method 4, (fit reduced data but include pseudo data-

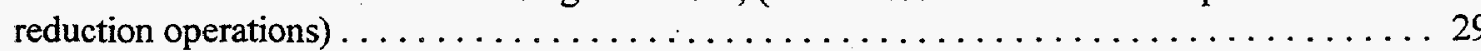

15 Fission cross section for ${ }^{241} \mathrm{Am}$ using Method 5, implicit data covariance (fit adjusted

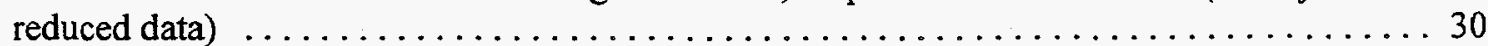




\section{LIST OF TABLES}

Table

Page

$1 \quad$ Penetrability (penetration factor) $P_{l}$ level shift factor $S_{l}$ and potential scattering phase shift $\phi_{l}$ for orbital angular momentum $l$, center of mass momentum $k$, and channel radius $a$, with $\rho=k a$.

2 The output parameter correlation matrix (times 100) found from fitting the artificial data

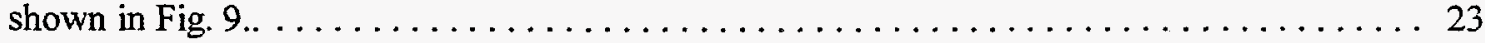

3 Comparison of resonance parameter values found using the methods described in the text. . . 27

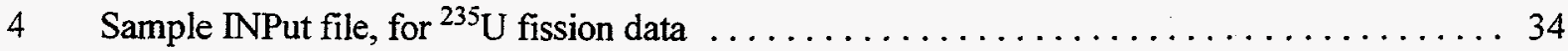

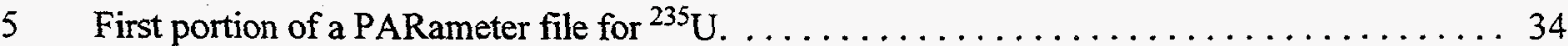

6 Some of the resonance parameters and data-reduction parameters from a PARameter file for

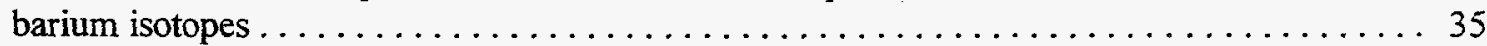

7 MULTI-style DATa file (relative uncertainties) $\ldots \ldots \ldots \ldots \ldots \ldots \ldots \ldots \ldots \ldots \ldots$

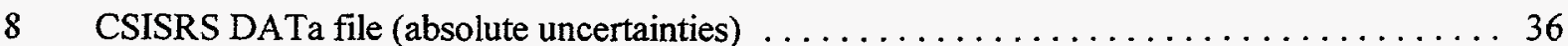

9 Worksheets for keeping track of details of experiments $\ldots \ldots \ldots \ldots \ldots \ldots \ldots \ldots$ 



\section{PREFACE}

This work was initially presented as a series of lectures at the Workshop on Nuclear Reaction Data and Nuclear Reactors-Physics, Design and Safety held February 23 through March 27, 1998, at the International Centre for Theoretical Physics, Trieste, Italy, and subsequently published in the Proceedings of that workshop. The lectures (and hence this report) are intended to serve as an introduction to the theory underlying the analysis of neutron-induced cross-section data in the resolved resonance region. Important features of the analysis process are discussed, both in general and with respect to the author's analysis code SAMMY; guidance is given for performing a complete evaluation in the resonance region. Also discussed are extensions into other topics that are not generally considered a part of this analysis process (group cross sections, integral quantities, unresolved-resonance region).

N. M. Larson 



\section{ACKNOWLEDGMENTS}

This work would not be possible without the considerable contributions from SAMMY users, too numerous to mention; these contributions include (but are not limited to) error discovery, suggestions for improvements, and ideas for extensions to the code. The author particularly wishes to thank Luiz Leal, ORNL, for his support, encouragement, and suggestions, and especially for his assistance in writing Sect. 7 of this paper. 


\title{
INTRODUCTION TO THE THEORY AND ANALYSIS \\ OF RESOLVED (AND UNRESOLVED) \\ NEUTRON RESONANCES VIA SAMMY
}

\author{
N. M. Larson
}

\begin{abstract}
Neutron cross-section data are important for two distinct purposes: First, they provide insight into the nature of matter, thus assisting in our understanding of fundamental physics. Second, they are needed for practical applications (e.g., for calculating when and how a reactor will become critical, or how much shielding is needed for storage of nuclear materials, and for medical applications). Neutron cross section data in the resolvedresonance region are generally obtained by time-of-flight experiments, which must be carefully analyzed if they are to be properly understood and utilized. In this paper, important features of the analysis process are discussed, with emphasis on the particular techniques used in the analysis code SAMMY. Other features of the code are also described; these include such topics as calculation of group cross sections (including covariance matrices), generation and fitting of integral quantities, and extensions into the unresolved-resonance region and higher-energy regions.
\end{abstract}

\section{Overview}

The interaction of neutrons with nuclei is a topic of great interest and importance in many areas of physics: for understanding of fundamental nuclear physics, for nuclear astrophysics studies, for reactor design and safety considerations, and for medical applications, to name just a few. For all applications, basic experimental nuclear data are needed; for a given neutron energy, the cross section for a particular reaction (i.e., the probability that a particular reaction will take place) must be known or estimated.

Because nuclear theory has not developed to the stage where resonance-region cross sections can be calculated from first principles, the cross sections must be obtained from measured data; nevertheless, measured data are not, of themselves, directly useful. What is more useful is the parameterization of that data in terms of scattering theory [i.e., in terms of the position (in energy) and of the widths of the individual resonances]. There are two primary reasons why the parameterization is more useful than the measured data: The first is the overabundance of information in the measured data, the second is the lack of information.

First, there is too much information: Tabulated values for cross section vs energy require hundreds of thousands of numbers; angular distributions require even more. Human minds are not equipped to interpret so many numbers; even with modern computers it is easier and more practical to store and manipulate a few resonance parameters rather than to keep track of so many numbers.

Second, there is not enough information: Extrapolations into the unresolved-resonance region require knowledge of statistical properties of the nucleus, such as average level spacings or strength functions. For many calculations, energies between and beyond the measured values may be needed. Some calculations require knowledge of the cross-section values at different experimental conditions, for which no data exist; the parameterized cross sections can be extended mathematically for different temperatures (Doppler broadening) or geometries (self-shielding calculations, multiplescattering effects, etc.), but the experimental data themselves cannot. Estimations of cross sections (for those nuclides for which no experimental data exist) can be made much more readily from 
systematics than from raw data. (Additional discussions on this topic are given in papers by Fröhner. ${ }^{1,2}$ )

At relatively low energies, individual peaks (resonances) can be seen in the energy-dependent cross sections; this energy region is labeled the "resolved-resonance region." At higher energies, the natural widths of the resonances are comparable to their spacing, so it is not possible to separate one peak from another; this is the "unresolved-resonance region." At even higher energies, the very concept of resonance is inappropriate; this region is referred to as the "high-energy region." Our primary concern in this paper is the resolved resonance region, in which the experimental data (cross sections vs energy) may be parameterized in terms of resonance energies, widths, and other quantities. The important features of data analysis in the resolved-resonance region are discussed, with particular emphasis on techniques used in the author's analysis code SAMMY. ${ }^{3}$ (Another approach to the same topic is given in Ref. 4.) Here we begin with a description, in general terms, of the kinds of experimental data that are available for analysis.

\section{Experimental Data}

\subsection{Neutron Time-of-Flight Experiments}

Neutron time-of-flight experiments are used to measure energy-dependent neutron cross sections at facilities such as the Oak Ridge Electron Linear Accelerator (ORELA) in Tennessee, the Geel LINAC (GELINA) in Belgium, or the Gaerttner LINAC at Rensselaer Polytechnic Institute in New York. These accelerators produce beams of electrons, which are then directed onto a target (tantalum at ORELA, uranium at GELINA); the resulting interaction produces neutrons, which exit outward in all directions. Shielding is used to collimate the beam by stopping neutrons traveling in an unwanted direction. For purposes of this report, the entire operation described above can be viewed as a "black box"; only the resulting neutron beam is of interest here.

Neutrons produced as described above are used as probes to study nuclei. Samples of the material to be studied are placed in the beam line, where neutrons interact with nuclei in the sample. Detectors, placed in or near the beam line (depending upon the type of data to be collected), are used to count the particles" that reach them. The schematic shown in Fig. 1 depicts a typical setup for a transmission experiment, in which the quantity to be determined is the ratio of the number of neutrons impinging on the sample to the number of neutrons leaving the sample; this quantity is directly related to the total cross section. Other types of differential (i.e., energy- and/or angle-dependent) measurements can also be performed with time-of-flight techniques, with appropriate modifications in the experimental setup.

The electron beam (and hence the neutron beam) is pulsed, with the beam "on" for, typically, a few nanoseconds, and "off" for several milliseconds. The detector (with associated electronics) records not merely the total number of arriving

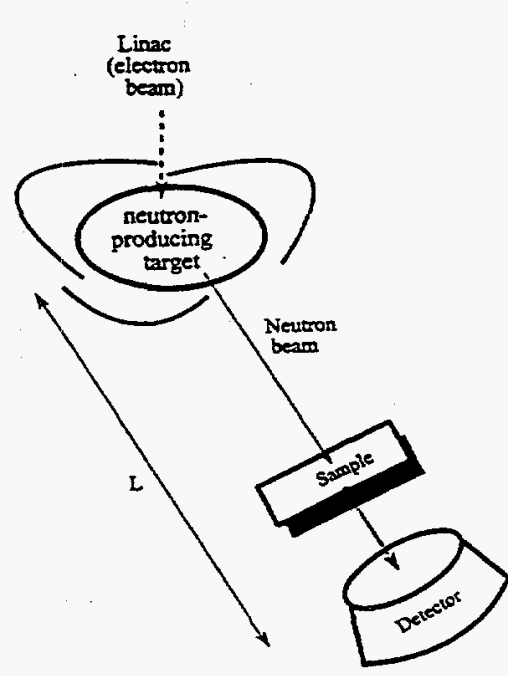

Figure 1: Schematic of a neutron timeof-flight transmission experiment. 
neutrons but also the time at which the neutrons arrive; that is, the raw data consist of counts vs time. The experimenter then must convert from counts to cross section, and from time to energy. This data reduction (i.e., the process for converting counts to cross section) involves detector dead-time corrections, background subtraction, normalization, and a host of other operations (of which more will be discussed later). Conversion from time to energy is done via the formula

$$
E=\frac{m}{2}\left(\frac{L}{\left(t-t_{0}\right)}\right)^{2}
$$

for which the path length $L$ has been measured precisely, $t_{0}$ represents the time at which the neutron leaves the target, and $t$ is the time at which the neutron (or other particle) reaches the detector.

\subsection{Types of Data}

Data obtained in neutron time-of-flight experiments are "differential" data, denoting the energydependent (as opposed to energy-integrated) nature of the measurement. Various types of differential data can be measured, with varying levels of accuracy and ease. Those types that can be analyzed within SAMMY are transmission data, total cross section, elastic cross section (both angle-dependent and angle-integrated), fission, inelastic scattering, capture, absorption, eta, and selfindication data; other data types will be added as the need arises.

Examples $^{5}$ of transmission measurements are shown in Fig. 2; examples of total, fission, and capture data are shown in Fig. 3. Note that the figures show only a small portion of the data from these experiments; the ${ }^{235} \mathrm{U}$ transmission measurement is from thermal to $2.25 \mathrm{keV}$, so only about $2 \%$ of the data are shown in Fig. 2.

In addition to these differential data, SAMMY can also calculate and fit to a variety of types of integral data: thermal cross sections, average integrals, Maxwellian average, $K 1$, Westcott's gfactor, $\alpha$, resonance integral, and reaction rates. Integral data are discussed more fully in Sect. 4.

\section{Analysis of Differential Data}

Analysis of neutron cross-section data in the resolved-resonance region has three distinct aspects, each of which must be included in any analysis code: First, an appropriate formalism is needed for generating theoretical cross sections. Second, a plausible mathematical description must be provided for every experimental condition that affects the values of the quantities being measured. Third, a fitting procedure must be available to determine the parameters values which provide the "best" fit of theoretical to experimental numbers.

The first of these aspects has been well understood for forty years; see especially the definitive paper by Lane and Thomas. ${ }^{9}$ The third is generally well understood, though often not done properly. The second, however, can never be accomplished perfectly; instead, what one must do is to design and implement progressively better approximations for whatever physical effects may be relevant to a particular experiment. In the following subsections, each of these three aspects is discussed individually. 


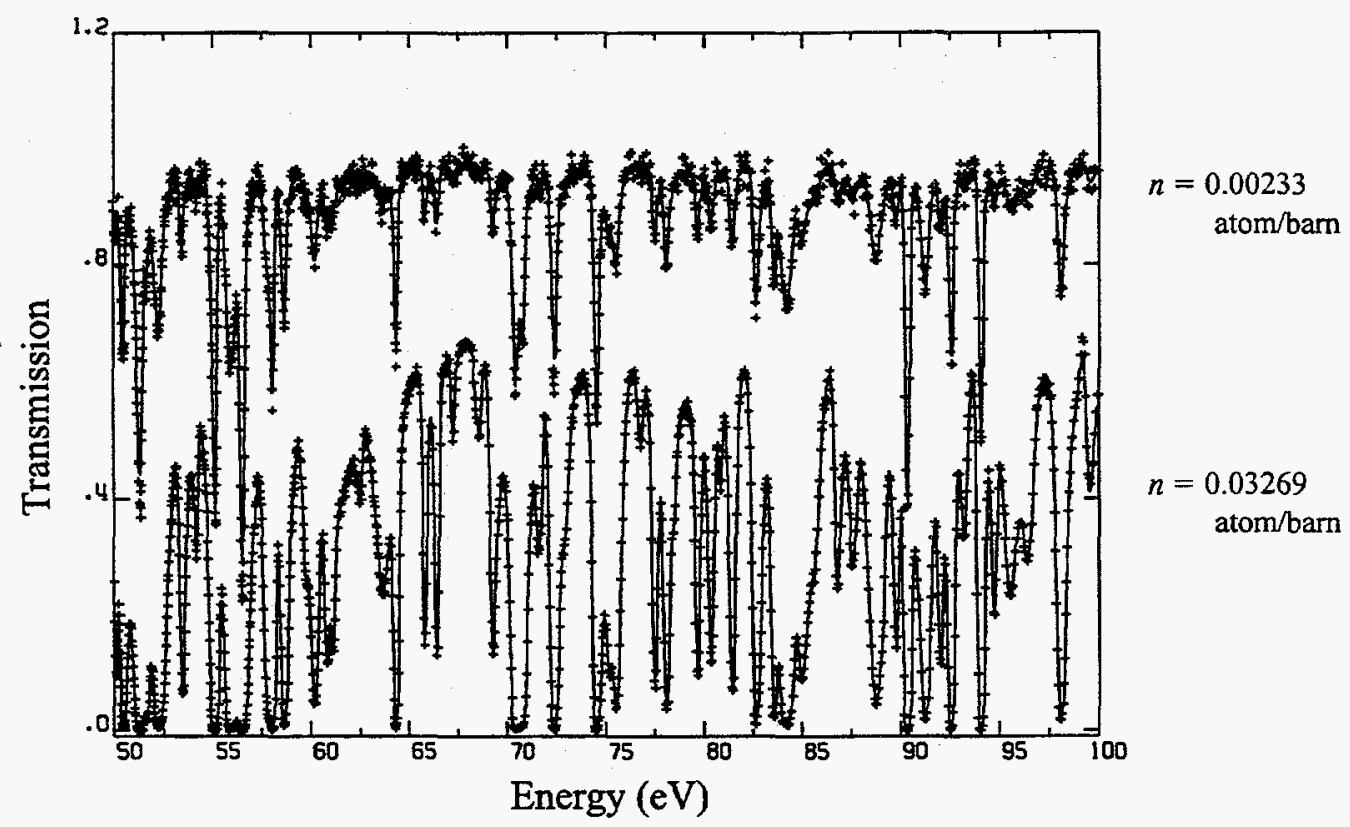

Figure 2: ${ }^{235} \mathrm{U}$ experimental (crosses) and calculated (solid line) neutron transmission data ${ }^{5}$ in the energy range from 50 to $100 \mathrm{eV}$. Results of the two sample thickness transmission experiments of Harvey et al. ${ }^{6}$ are displayed.

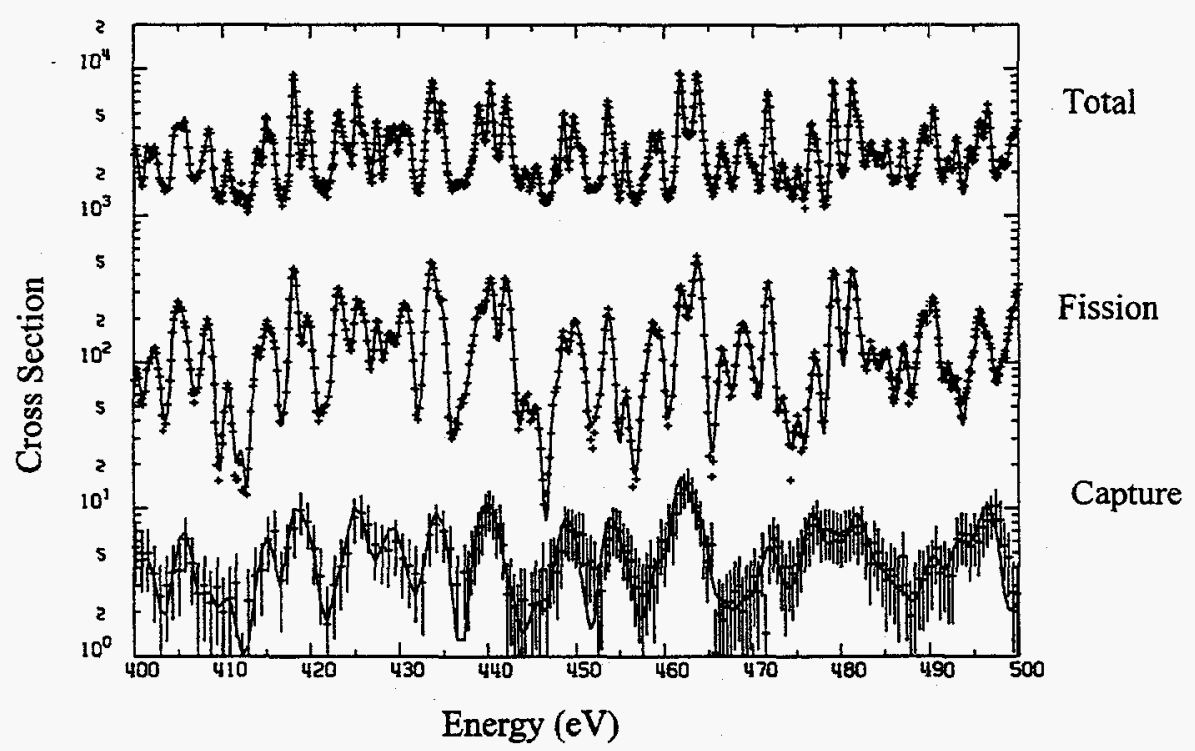

Figure 3: ${ }^{235} \mathrm{U}$ experimental (crosses) and calculated (solid curve) total cross section, fission cross section, and capture cross section in the energy range from 400 to $500 \mathrm{eV}$. The top curve is Harvey et al. total cross-section data $^{6}$ (multiplied by 100 ), the center curve is Weston fission data (multiplied by 10 ), and the bottom curve is deSaussure capture cross-section data. ${ }^{8}$ 


\subsection{Formalism for Calculation of Cross Section}

In addition to the detailed treatise by Lane and Thomas, ${ }^{9}$ a wealth of reference material is available to the student of scattering theory. The text by Foderaro ${ }^{10}$ provides a more elementary introduction to the subject. Two publications by Fröhner are highly recommended resources: The first ${ }^{1}$ is based on lectures presented at the ICTP Winter Courses on Nuclear Physics and Reactors, 1978; this is a comprehensive and useful guide to applied neutron resonance theory. It includes a variety of topics: preparation of data, the various approximations to R-matrix theory, Doppler broadening, experimental complications, data-fitting procedures, and statistical tests. The second Fröhner paper ${ }^{2}$ is from the 1992 ICTP conference and is essentially an update of the first.

Scattering Theory, sometimes called Collision Theory or R-Matrix Theory, is a mathematically rigorous phenomenological description of what is actually seen in an experiment (i.e., the measured cross section). The theory is not a model of neutron-nucleus interaction, in the sense that it makes no assumptions about the underlying physics of the interaction. Instead it parameterizes the measurement in terms of (more-or-less) observable quantities, such as the interaction radii and boundary conditions, resonance energies and widths, and quantum numbers. The theory is mathematically correct, in that it is analytic, unitary, and rigorous; nevertheless, in practical applications the theory is always approximated in some fashion.

Scattering theory is based on the following assumptions ${ }^{9}$ : (1) the applicability of nonrelativistic quantum mechanics; (2) the absence or unimportance of all processes in which more than two product nuclei are formed; (3) the absence or unimportance of all processes of creation or destruction; and (4) the existence of a finite radial separation beyond which no nuclear interactions occur. [In practical applications three of these four assumptions may be violated in one degree or another: (1) The theory may be used for relativistic neutron energies, and Eq. (1) corrected for relativistic effects; nevertheless, nonrelativistic quantum mechanics is assumed. (2) A fission experiment with more than two final products may be treated as if the immediate result of the neutron-nuclide interaction were limited to two final products, at least one of which decays prior to detection. (4) Coulomb interactions can be treated specially.]

Scattering theory is couched in terms of channels; a channel is defined as a pair of (incoming or outgoing) particles, described by mass, charge, and spin quantum numbers. Mass refers to the atomic mass of the particles. In SAMMY, masses are expressed in atomic mass units (amu); the convention used in the Evaluated Nuclear Data Files $\left(E N D F^{11}\right.$ ) is "ratio to neutron." Charge is zero for neutrons, and therefore generally irrelevant for neutron-induced cross sections (though needed for outgoing fission products). Spin quantum numbers are defined as follows: The intrinsic spin of the incident neutron is $i=1 / 2$, and the parity is positive. The intrinsic spin of the nuclide is $I$ (integer or halfinteger), parity may be positive or negative. The relative orbital angular momentum of the neutronnuclide pair is $l$, parity is positive for even $l$ and negative for odd $l$. Intrinsic spins $i$ and $I$ are coupled vectorially to give channel spin $s$ (called $j$ instead of $s$ in the SAMMY users' manual ${ }^{3}$ ), then $s$ is coupled to $l$ to give the resonance spin $J$. Only $J$ and parity are conserved in an interaction.

A schematic depicting entrance and exit channels is shown in Fig. 4. Note that entrance channels can also occur as exit channels, but some exit channels (e.g., fission channels) do not occur as entrance channels. Two particles interacting are shown in the portion of Fig. 4 that is labeled "Internal Region"; here the particles are separated by less than the interaction radius $a$. 

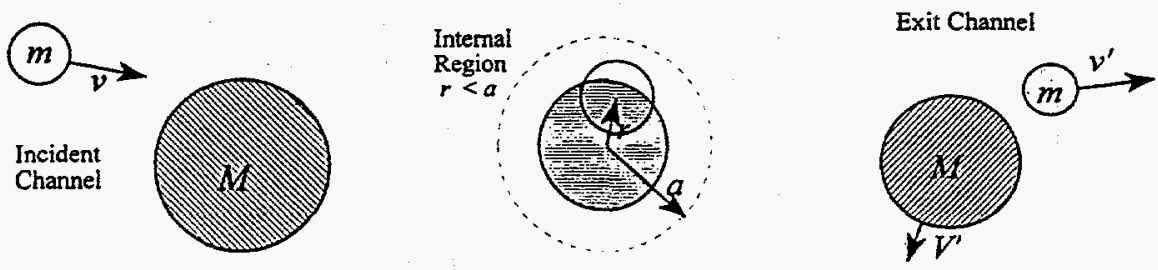

Figure 4: Schematic of entrance and exit channels as used in scattering theory. For the interior region $(r<a)$, no assumptions are made about the nature of the interaction.

The first step of the derivation is to parameterize the external wave function as

$$
\psi=e^{i k z}+f(\theta) e^{i k r} / r,
$$

so that the differential cross section can be written as

$$
\frac{d \sigma}{d \Omega}=|f(\theta)|^{2}
$$

Here $f$ has the form

$$
f(\theta)=\frac{1}{2 i k} \sum_{l}(2 l+1)[U-1] P_{l}(\cos \theta),
$$

which can be derived by expanding the general solution of Schrödinger's Equation for zero potential. The quantity $l$ in this equation is the orbital angular momentum referred to above. Subscripts $c$ and $c^{\prime}$, representing all quantum numbers for the initial and final channels, are suppressed in these equations.

The next step in the derivation is to expand the internal radial wave function in terms of eigenfunctions whose derivatives are zero at the boundary (i.e., at $r=a$ ). Equating the internal and external wave functions and derivatives at the boundary gives the scattering matrix $U$ as

$$
U_{c c^{\prime}}^{J}=\Omega_{l} W_{c c^{\prime}}^{J} \Omega_{l^{\prime}}
$$

with

$$
\Omega_{l}=e^{-i \phi_{l}},
$$

where $\phi_{l}$ is the potential phase shift (sometimes called the "hard sphere phase shift"). In matrix notation, with all indices suppressed, $W$ is given by

$$
W=P^{1 / 2}(I-R L)^{-1}\left(I-R L^{*}\right) P^{-1 / 2}
$$

with

$$
L=(S-B)+i P,
$$

where $P$ is the penetrability, $S$ is the shift factor, $B$ is the arbitrary boundary constant at the channel radius $a, I$ is the identity matrix, and $R$ is the R-matrix.

Exact expressions for $P_{l}, S_{l}$, and $\phi_{l}$ are found in the scattering theory derivation, and are shown in Table 1. The expressions are presented as functions of $\rho$, for 
Table 1: Penetrability (penetration factor) $P_{l}$ level shift factor $S_{l}$ and potential scattering phase shift $\phi_{l}$ for orbital angular momentum $l$, center of mass momentum $k$, and channel radius $a$, with $\rho=k a$.

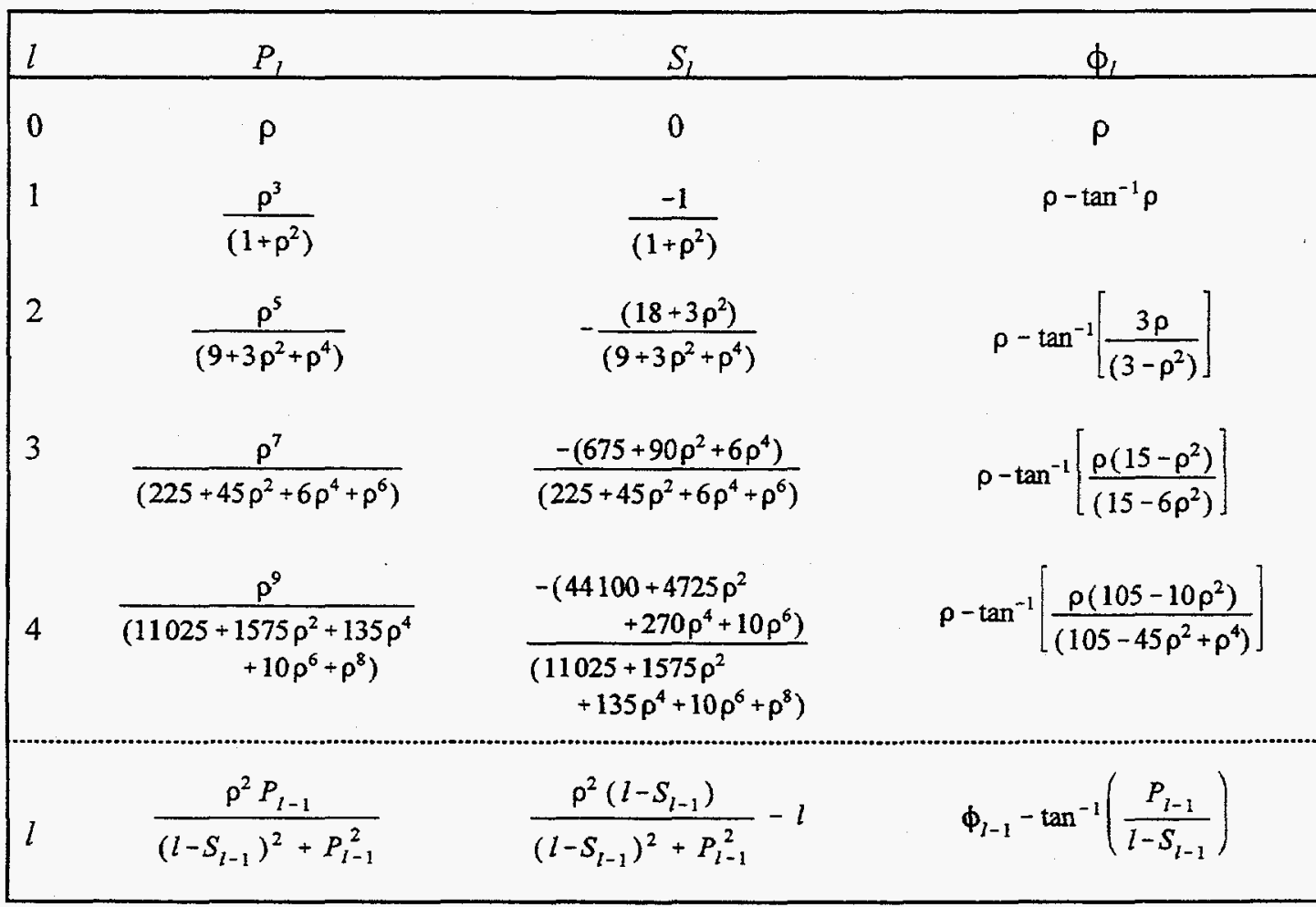

$$
\rho=k a,
$$

where $k$ is the center of mass momentum (often called wave number) for channel $c$, given by

$$
k^{2}=2 m\left(\frac{M}{M+m}\right)^{2} E,
$$

where $m$ is the neutron mass, $M$ is the nuclear mass, and $E$ is the energy of the neutron in the laboratory system.

The expression for the R-matrix used in Eq. (7) can appear in various guises, depending on the particular approximation that is to be used. In the exact $\mathrm{R}$-matrix theory, $R$ is in the form

$$
R_{c c^{\prime}}=\sum_{\lambda} \frac{\gamma_{\lambda c} \gamma_{\lambda c^{\prime}}}{E_{\lambda}-E}
$$

and the sum over $\lambda$ includes an infinite number of levels (i.e. resonances). (Limiting this to a finite number is the one approximation shared by all versions of R-matrix theory.) In this equation, the subscripts $c$ and $c^{\prime}$ represent any of the possible channels (neutron, capture, fission channels). The quantities $\gamma$ are called reduced-width amplitudes, and are related to the width for the respective channel via 


$$
\Gamma_{\lambda c}=2 P_{l} \gamma_{\lambda c}^{2}
$$

for neutron channels, and via

$$
\Gamma_{\lambda c}=2 \gamma_{\lambda c}^{2}
$$

for capture or fission channels.

One common approximation for the R-matrix is the result of neglecting the level-level interference for the capture channels; this is the Reich-Moore approximation, for which the R-matrix takes the form

$$
R_{c c^{\prime}}=\sum_{\lambda} \frac{\gamma_{\lambda c} \gamma_{\lambda c^{\prime}}}{E_{\lambda}-E-i \gamma_{\lambda \gamma}^{2}}
$$

Here the subscripts $c$ and $c^{\prime}$ represent only particle channels (i.e., non-capture channels).

\subsubsection{Implementation within SAMMY}

Equation (7) for $W$ can be rewritten in the form

$$
W=I+2 i P^{1 / 2} L^{-1}\left(L^{-1}-R\right)^{-1} R P^{1 / 2}=I+2 i X,
$$

where $X$ is defined by

$$
X=P^{1 / 2} L^{-1}\left(L^{-1}-R\right)^{-1} R P^{1 / 2},
$$

with $R$ as given by either Eq. (11) or Eq. (14). The total cross section can then be expressed in terms of $X$ as

$$
\sigma^{\text {total }}=\frac{4 \pi}{k^{2}} \sum_{J} g_{J} \sum_{\substack{\text { incident } \\ \text { channels } c}}\left(\sin ^{2}\left(\phi_{c}\right)+\cos \left(2 \phi_{c}\right) \operatorname{Im}\left(X_{c c^{\prime}}^{J}\right)-\sin \left(2 \phi_{c}\right) \operatorname{Re}\left(X_{c c}^{J}\right)\right),
$$

in which the "spin statistical factor" $g_{J}$ is defined as

$$
g_{J}=\frac{2 J+1}{(2 i+1)(2 I+1)} \text {. }
$$

The elastic cross section is given by

$$
\sigma^{\text {elastic }}=\frac{4 \pi}{k^{2}} \sum_{J} g_{J} \sum_{\substack{\text { incident } \\ \text { channels } c}}\left(\sin ^{2}\left(\phi_{c}\right)\left(1-2 \operatorname{Im}\left(X_{c c^{\prime}}^{J}\right)\right)-\sin \left(2 \phi_{c}\right) \operatorname{Re}\left(X_{c c}^{J}\right)\right),
$$

the fission (reaction) cross section by

$$
\sigma^{\text {reaction }}=\frac{\pi}{k^{2}} \sum_{J} g_{J} \sum_{\begin{array}{c}
\text { incident } \\
\text { channels c }
\end{array}} \sum_{\substack{\text { exit } \\
\text { channels } c^{\prime}}}\left|X_{c c^{\prime}}^{J}\right|
$$

and the capture cross section by

$$
\sigma^{\text {capture }}=\frac{\pi}{k^{2}} \sum_{J} g_{J} \sum_{\begin{array}{c}
\text { incident } \\
\text { channels } c
\end{array}}\left(\operatorname{Im}\left(X_{c c}^{J}\right)-\sum_{\begin{array}{c}
\text { all } \\
\text { channels } c^{\prime}
\end{array}}\left|X_{c c^{\prime}}^{J}\right|\right) .
$$

Other types of cross sections (e.g., inelastic, absorption) may similarly be defined in terms of $X$.

Within SAMMY, the "exact" formulation for the R-matrix, Eq. (11), is not yet available but will be implemented in SAMMY when time permits. The most rigorous formulation currently available 
within SAMMY is the Reich-Moore approximation, Eq. (14). In general, Reich Moore is indistinguishable from the exact formulation for most purposes; the only notable exception is for low-mass nuclei. (Gerry Hale's code uses the exact formulation; see Ref. 12.)

Also available within SAMMY are both the single-level and the multi-level Breit Wigner formulations; these are included for the sake of completeness, for comparison purposes, and because many of the evaluations in the nuclear data files ${ }^{13}$ were performed with Breit-Wigner formulae. However, it is strongly recommended that only Reich Moore be used for new evaluations, for several reasons: Multilevel Breit Wigner (MLBW) is often inadequate; single-level Breit Wigner is almost always inadequate. When it is correct, MLBW gives identical results to Reich Moore. "Ease of Programming" is no longer a good excuse for using MLBW, since the programming has already been accomplished. Similarly, a slow computer is no longer a good excuse, since modern computers can handle the more rigorous formulae quite readily.

\subsection{Mathematical Description of Experimental Effects}

Even though scattering theory provides a mathematically rigorous description of the interaction between a neutron and a nucleus, nevertheless it alone gives neither a complete nor an accurate picture of the quantities that are measured in an actual experiment. It is impossible to measure the interaction of a single neutron with a single nucleus, in the absence of all other effects. Instead, an experiment includes all the complexity resulting from being part of the real world: The neutron beam consists of many neutrons, not just one. The sample is of finite size (i.e., there are many nuclei, not just one). The sample may include many different kinds of nuclei (isotopes, chemical compounds, and/or impurities). Because it is not possible to achieve absolute zero temperature, the sample nucleus is moving rather than sitting still in the laboratory. The time at which the neutron leaves the neutron-producing target is not known exactly, but only within the neutron burst width. The distance that the neutron must travel is not known exactly. The neutron-producing target is finite size, so the position from which the neutron begins its flight is known only within the target thickness. The detector is of finite size, so the position at which the neutron is detected is also not known exactly.

All these conditions affect the number of neutrons that are tallied for a given time-channel in a given experiment. Consequently, the analysis procedure must modify the cross sections generated by the scattering theory, using mathematical models of these experimental effects, in order to provide meaningful comparisons with the measured quantities.

In the following sections, many of these effects are described individually, and the corresponding mathematical models are presented.

\subsubsection{Doppler broadening}

Doppler broadening refers to the average over the thermal motion of the particles within the sample. Nuclei within the sample exhibit random thermal motion; in macroscopic terms, the temperature is not absolute zero. The effect of this thermal motion on the cross section is often dramatic, as shown in Fig. 5.

Two options (with variations of each) have been used to describe the finite-temperature effect. The first assumes the sample is a gas (free-gas model), and the second assumes the sample is a solid 


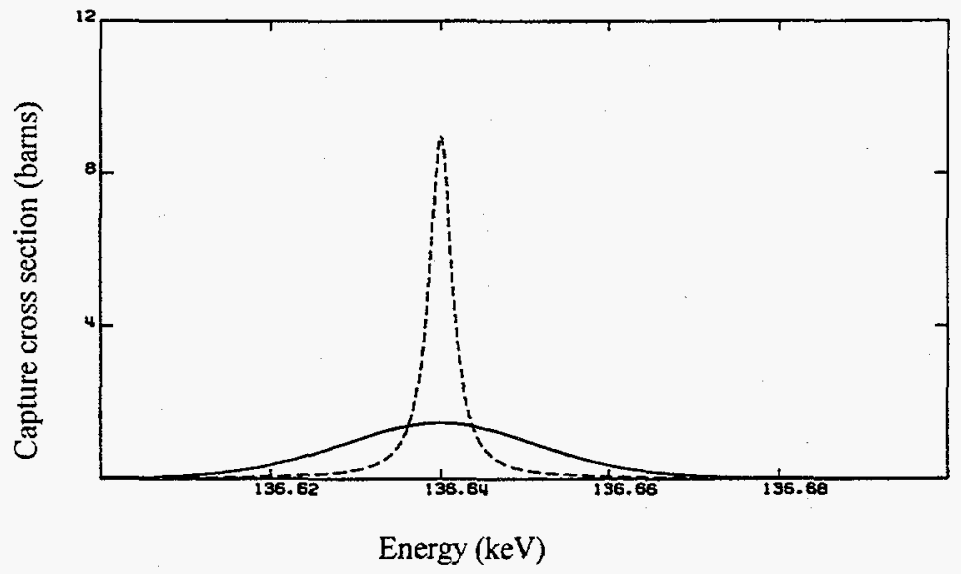

Figure 5: Capture cross section for one resonance in ${ }^{58} \mathrm{Ni}$, without Doppler broadening (dashed curve) and with Doppler broadening at $300 \mathrm{~K}$ via the free-gas model (solid curve).

(crystal-lattice model). Somewhat counter-intuitively, the free-gas model has been seen to work well in most physical situations, even for very heavy nuclei such as lead or uranium. The crystal lattice model is more realistic, and hence more mathematically complex; in certain physical situations it is expected to provide a more accurate picture of the Doppler effect.

Historically, most early analysis and data-processing codes used the high-energy Gaussian approximation (HEGA) to the free-gas model (FGM), because of its convenient analytical properties: The HEGA, when mathematically convoluted with a Breit-Wigner formulation for the cross sections, results in analytic expressions for the Doppler-broadened cross section, in terms of the psi $(\psi)$ and chi $(\chi)$ functions. Prior to the advent of modern computers, this analytic feature was a major advantage; it permitted otherwise-impossible calculations to proceed.

With modern computational capabilities, however, there is no longer any unique advantage to using the HEGA. Indeed, today one should use only the FGM and not the HEGA: First, the original advantage of HEGA is no longer relevant, because the analytic formulae are applicable only for the Breit-Wigner approximation, which, as we have seen, is often inappropriate. Second, the approximations involved in generating the HEGA are invalid at low energies; differences between HEGA and FGM are noticeable in calculations even at relatively high energies; details are given in Ref. 14. (Such differences at high energies are not likely to be visible experimentally, however, except for very high-resolution experiments.)

\subsubsection{Derivation of Free-Gas Model}

Derivations of the free-gas model from first principles have been published by many sources $1,2,14,15,16$ and will not be repeated in detail here. Instead the process will be outlined and the results presented.

Assume that a parallel beam consisting of monoenergetic particles with lab velocity $\vec{v}$ impinges upon a sample. Nuclei in the sample have velocities $\vec{W}$, distributed in such a way that $p(\vec{W}) d^{3} W$ is the fraction with velocities in a small, three-dimensional region $d^{3} W$ around $\vec{W}$. The densities of the beam and sample particles are, respectively, $\rho_{1}$ and $\rho_{2}$. The unbroadened cross section is denoted $\sigma(\mathrm{E})$, and $\bar{\sigma}\left(m v^{2} / 2\right)$ is the effective or Doppler-broadened cross section for incident particles with speed $v$ [laboratory energy $m v^{2} / 2$ ]. Thus the number of reactions occurring per unit time and unit volume is 


$$
\rho_{1} \rho_{2} \int d^{3} W p(\vec{W})|\vec{v}-\vec{W}| \sigma\left(m|\vec{v}-\vec{W}|^{2} / 2\right) \equiv \rho_{1} \rho_{2} v \bar{\sigma}\left(m v^{2} / 2\right)
$$

Assume that the sample nuclei have the same velocity distribution as the atoms of an ideal [monatomic] gas (i.e., the Maxwell-Boltzmann distribution),

$$
p(\vec{W}) d^{3} W=\frac{1}{\pi^{3 / 2}} \exp \left(-\frac{W^{2}}{u^{2}}\right) \frac{d^{3} W}{u^{3}},
$$

with $u$ defined by

$$
\frac{M}{2} u^{2}=k T
$$

where $M$ is the nuclear mass, $k$ is Boltzmann's constant, and $T$ is the temperature. Then the Doppler-broadened cross section is given by

$$
v \bar{\sigma}\left(m v^{2} / 2\right)=\frac{1}{\pi^{3 / 2} u^{3}} \int_{\text {all } \bar{W}} d^{3} W \exp \left(-\frac{W^{2}}{u^{2}}\right)|\vec{v}-\vec{W}| \sigma\left(m|\vec{v}-\vec{W}|^{2} / 2\right) .
$$

This expression can be simplified by changing the integration variable from $\vec{W}$ to $\vec{w}=\vec{v}-\vec{W}$, and choosing spherical coordinates. Note that several lines of algebra have been omitted in this derivation; complete details are available in Ref. 14.

$$
\begin{aligned}
v \bar{\sigma}\left(\frac{m v^{2}}{2}\right) & =\frac{1}{\pi^{3 / 2} u^{3}} \int_{\text {all } \bar{w}} d^{3} w \exp \left(-\frac{\left(v^{2}-2 v w \cos \theta+w^{2}\right)}{u^{2}}\right) w \sigma\left(\frac{m w^{2}}{2}\right) \\
& =\frac{1}{v \sqrt{\pi} u} \int_{0}^{\infty} d w w^{2} \sigma\left(\frac{m w^{2}}{2}\right)\left[\exp \left(-\frac{(v-w)^{2}}{u^{2}}\right)-\exp \left(-\frac{(v+w)^{2}}{u^{2}}\right)\right]
\end{aligned}
$$

\subsubsection{Implementation within SAMMY}

The free-gas model (FGM), as implemented within SAMMY, relies on a simple mathematical transformation to put Eq. (26) into a more concise form. If a "pseudo cross section" $s$ is defined as

$$
\begin{aligned}
s(w) & =\sigma\left(m(w)^{2} / 2\right) & & \text { for } w>0 \\
& =-\sigma\left(m(-w)^{2} / 2\right) & & \text { for } w<0,
\end{aligned}
$$

then the free-gas model of Doppler broadening can be written as

$$
\bar{\sigma}\left(\frac{m v^{2}}{2}\right)=\frac{1}{v^{2} \sqrt{\pi} u} \int_{-\infty}^{\infty} d w w^{2} s(w) \exp \left(-\frac{(v-w)^{2}}{u^{2}}\right) .
$$

In this form it is clear that an unbroadened cross section inversely proportional to velocity is transformed into itself, since, if $s(w)=\sigma_{0} v_{0} / w$, then Eq. (28) becomes

$$
\bar{\sigma}\left(\frac{m v^{2}}{2}\right)=\frac{\sigma_{0} v_{0}}{v^{2} \sqrt{\pi} u} \int_{-\infty}^{\infty} d w w \exp \left(-\frac{(v-w)^{2}}{u^{2}}\right)=\frac{\sigma_{0} v_{0}}{v}
$$

Three versions of Doppler broadening are available for SAMMY users. The one whose use is recommended in virtually all cases is the free-gas model, based on an implementation of Eq. (28); 
see pages $92 q$ through $92 \mathrm{r}$ of the SAMMY users' manual ${ }^{3}$ for details. The required integrations are performed numerically, using an appropriate grid in velocity-space. This method is relatively accurate and efficient for all energies, even when there is structure in the cross section, so long as the grid is sufficiently dense. (SAMMY users are encouraged to test the accuracy of their calculations by performing a run with increased density of points.)

A second implementation of the free-gas model, based on work by Leal and Hwang, ${ }^{15}$ is also available in SAMMY (pages 92a-92c of the users' manual). In this formulation the Dopplerbroadened cross section is the solution of the partial differential equation that has the same form as the 1-D, time-dependent heat equation. This implementation requires a uniform grid in velocity space, and thus is efficient only when the cross section is slowly varying (i.e., when there are no resonances).

The third implementation of Doppler broadening in SAMMY is the HEGA, for which again integrations are performed numerically using an appropriate grid in energy-space (page $61 \mathrm{ff}$ of the users' manual). This was originally the only Doppler broadening in many analysis codes (including SAMMY), but its use is now discouraged.

The crystal-lattice model may be important for some low-energy cross sections, and therefore will soon become the fourth option for Doppler broadening in SAMMY. Interested readers are referred to the report by Meister et al. ${ }^{17}$ for details on this model.

\subsubsection{SAMMY's Integration Methodology}

The integrations needed in order to evaluate Doppler-broadened cross sections (e.g., via Eq. (28) for the free-gas model) require the use of numerical integration schemes. In the next section it will be seen that the same is true of resolution broadening. Details about the integration scheme are given in Sect. IV.B, page 73ff, of the SAMMY users' manual, ${ }^{3}$ and are summarized here.

It is necessary to evaluate an integral of the form

$$
f\left(x_{i}\right)=\int_{E \min }^{E \max } \bar{f}\left(x^{\prime}\right) B\left(x_{i}, x^{\prime}\right) d x^{\prime},
$$

where $\left\{x_{i}\right\}$ is a predetermined set of grid points (in energy-space, velocity-space, or for whatever variable is appropriate) on which we wish to know the broadened values $f\left(x_{i}\right), \bar{f}\left(x^{\prime}\right)$ are the unbroadened theoretical values (for cross section, transmission, etc.), $B\left(x_{i}, x^{\prime}\right)$ is the Doppler- or resolution-broadening function, and the limits $E \min =0$ and $E \max =\infty$ (although in practice a smaller range is used). This integration is performed by transforming from a continuous integral to a discrete summation, by choosing points $\left\{x_{j}{ }^{\prime}\right\}$ and associated weights $\left\{W_{j}\right\}$ such that

$$
\int_{a}^{b} \bar{f}\left(x^{\prime}\right) B\left(x_{i}, x^{\prime}\right) d x^{\prime}=\sum_{j} \bar{f}\left(x_{j}{ }^{\prime}\right) B\left(x_{i}, x_{j}{ }^{\prime}\right) W_{j},
$$

where the approximation is exact if the integrand is a polynomial of specified degree. 
The choice of "auxiliary grid" $\left\{x_{j}{ }^{\prime}\right\}$ is somewhat arbitrary. SAMMY's choice (described in more detail on pages 79-80 in the users' manual) is as follows: Start with the experimental grid (converted from energy to velocity, if necessary). Add extra points between each point in grid (optional). Add points to the extremities (i.e., outside data range) so that the end-points can be broadened. Add enough points to adequately describe each resonance. Test the resulting grid to be sure that spacings do not vary too wildly among neighboring points.

Most integrations in SAMMY use the four-point progressive interpolation method of Mintz and Jordan ${ }^{18}$ to determine the weighting factors $W_{j}$. This interpolation method was used in the code MULTI, ${ }^{19}$ which was the precursor to SAMMY; numerous tests throughout the years have shown that this method appears sufficiently fast, accurate, and efficient that no change has been deemed necessary.

With SAMMY, it is the user (and not the code) who has primary control over the accuracy of the integrated values. A user should not assume that integrated values will automatically be as accurate as possible; at some time during an analysis, the user should add extra points to the auxiliary grid to test whether computations are sufficiently accurate.

\subsubsection{Resolution Broadening}

Resolution broadening is "smearing" of the cross section as a function of energy, and is caused by a variety of phenomena such as the spread in neutron burst width, the finite size of the neutronproducing target, the finite size of the time-of-flight channel width, and the finite size of the detector.

Unlike Doppler broadening, for resolution broadening there is no "standard" or "best" function that is used almost universally. Each analysis code has its own version of resolution broadening, often with specific formulations for specific experimental sites or setups.

Within SAMMY, resolution broadening can be described by one of three distinct methods, each with its own particular strengths. The earliest (and computationally simplest) of these is a Gaussian function (with or without an exponential tail). The second, called the Oak Ridge Resolution Function, is the convolution of four realistic components. The third is a similar convolution of realistic components relevant to the RPI Linac. A fourth resolution-broadening function is in initial planning stages; this function will be defined numerically rather than analytically.

\subsubsection{Gaussian and/or Exponential Resolution-Broadening Function}

The original resolution function available in SAMMY, borrowed directly from the precursor code MULTI, ${ }^{19}$ is a Gaussian and/or exponential function (pages 63-80 of the SAMMY users' manual). The Gaussian function is a combination of three components: a square function of (flight-path) length, a Gaussian in time (representing the burst width), and another square function in time (representing the channel width). To combine these three components, the two square functions are represented by their "equivalent" Gaussian functions (i.e., by the Gaussian functions with the same means and standard deviations). Values required for input to SAMMY are (1) the full width of a square distribution in length, (2) the full width at half maximum of the Gaussian burst width, and (3) the full width of the square time-channel (which may be different for different energy ranges).

Another option for this resolution function is the exponential function, or the Gaussian plus exponential, which provides an asymmetric component to the resolution function. 


\subsubsection{Oak Ridge Resolution Function}

A realistic resolution function (pages 92e-920 of the SAMMY users' manual; see also Ref. 20 ) has been developed for use primarily with data from the ORELA, although it may also be useful for data taken elsewhere. This function is the convolution of four components, one each to represent the electron burst width, the neutron source, the time-of-flight channel width, and the detector. Each component is described mathematically as a function of either time $t$ or flight-path-length $\ell$, then converted to a function of $t$ (for fixed $E$ ) using the formula

$$
E=\frac{m}{2} \frac{\ell^{2}}{t^{2}} \text {. }
$$

The "complete" resolution function is the convolution of the four individual pieces:

$$
I(t)=\int I_{1}\left(t-t_{1}\right) d t_{1} \int I_{2}\left(t_{1}-t_{2}\right) d t_{2} \int I_{3}\left(t_{2}-t_{3}\right) d t_{3} I_{4}\left(t_{3}\right)
$$

Integrations are analytical where possible (to maximize speed and accuracy), and numerical where necessary.

The components of the Oak Ridge Resolution Function are as follows:

1. The electron burst width is taken to be a square function in time,

$$
I_{1}(t)=\left\{\begin{array}{cl}
1 / p & \text { for } 0<t<p \\
0 & \text { otherwise }
\end{array}\right.
$$

where $p$ is the burst width in nanoseconds.

2. For the neutron source, there are two possibilities:

2a. The ORELA Water Moderator is assumed to be a $\chi^{2}$ distribution with $2(m+1)$ degrees of freedom, where $m=4$ or 5 . That is,

$$
I_{2 a}^{\prime}(\ell)=\frac{\ell^{m}}{m ! \Lambda^{m+1}} \exp \left(-\frac{\ell}{\Lambda}\right),
$$

where $\ell$ is the flight-path-length variable, and moderation distance $\Lambda$ is a mean free path. The value of $\Lambda$ varies with energy as

$$
\Lambda=\Lambda_{0}+\Lambda_{1} \ln (E)+\Lambda_{2}(\ln (E))^{2}
$$

2b. The second possibility for the neutron source is the tantalum target, for which the resolution function is a bit more complicated:

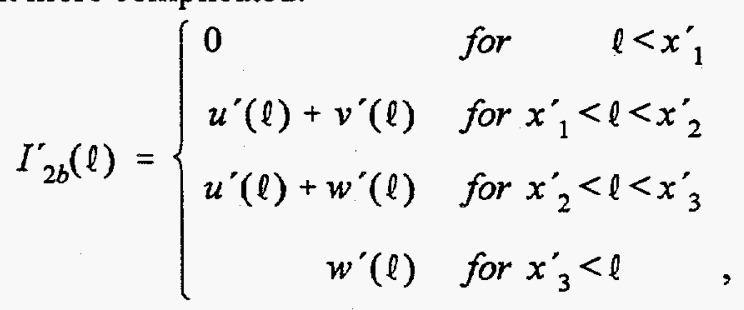

where the functions $u^{\prime}(\ell), v^{\prime}(\ell)$, and $w^{\prime}(\ell)$ are defined as 


$$
\begin{array}{ll}
u^{\prime}(\ell)=N^{\prime} \exp \left\{-\epsilon^{\prime 2}\left(\ell-x_{0}^{\prime}\right)^{2}\right\} & \text { for } x_{1}^{\prime}<\ell<x_{3}^{\prime}, \\
v^{\prime}(\ell)=\frac{N^{\prime} \alpha^{\prime} \ell}{x_{2}^{\prime}} \exp \left\{-\beta^{\prime}\left(\ell-x_{2}^{\prime}\right)\right\} & \text { for } x_{1}^{\prime}<\ell<x_{2}^{\prime},
\end{array}
$$

and

$$
w^{\prime}(\ell)=N^{\prime} \alpha^{\prime} \exp \left\{-\beta^{\prime}\left(\ell-x_{2}^{\prime}\right)\right\} \quad \text { for } x_{2}^{\prime}<\ell \text {. }
$$

3. The time-of-flight channel width is described as a square function in time, that is,

$$
I_{3}(t)=\left\{\begin{array}{cl}
1 / c & \text { for } 0<t<c \\
0 & \text { otherwise }
\end{array},\right.
$$

in which the channel width $c$ may be different for different energy-regions.

4. For the detector component of the resolution function, there are again two possibilities:

4a. The first is for the NE110 Detector, for which the resolution function is taken to be

$$
I_{4 a}^{\prime}(\ell)= \begin{cases}\Delta \exp (-\lambda \sigma \ell) & \text { for } 0<\ell<\delta \\ 0 & \text { otherwise } .\end{cases}
$$

Here parameter $\delta$ is the thickness of the detector, $\lambda$ is the number of molecules per mm.b of detector $(0.0047$ for NE1 10), and $\sigma(E)$ is the total cross section for the detector material $\left(\mathrm{CH}_{1.104}\right.$ for NE1 10). Parameter $\Delta$ is a normalization found from $\int I_{4 a}^{\prime}=1$, that is,

$$
\Delta=\frac{\lambda \sigma}{1-\exp (-\lambda \sigma \delta)}
$$

4b. The second option is for the Lithium Glass Detector, for which the resolution function is given by

$$
I_{4 b}(t)=\left\{\begin{array}{ll}
D g & \text { for } 0<t<d \\
D \exp (-f(t-d)) & \text { for } d<t
\end{array},\right.
$$

where $g$ is a constant, and $D$ is chosen to give $\int I_{4 b}^{\prime}=1$, that is,

$$
D=\frac{f}{1+g f d} \text {. }
$$

\subsubsection{RPI Resolution Function}

A second realistic resolution function (pages $92 \mathrm{~s}$ to $92 \mathrm{v}$ of Revision 4 of the SAMMY users' manual, ${ }^{21}$ see also Ref. 22) has been developed for use with data from the Gaerttner LINAC at Rensselaer Polytechnic Institute; this, too, may be useful for data taken on other machines as well. This function is the convolution of three components, one each to represent the electron burst width, the neutron source plus detector combined, and the time-of-flight channel width. Each component is described mathematically as a function of time-of-flight $t$. The "complete" resolution function is the convolution of the three individual pieces,

$$
I(t)=\int I_{1}\left(t-t_{1}\right) d t_{1} \int I_{2}\left(t_{1}-t_{2}\right) d t_{2} I_{3}\left(t_{2}\right) .
$$


Again, integrations required by the convolution process are analytical where possible (to maximize speed and accuracy), and numerical where necessary.

Components of the RPI Resolution Function are as follows:

1. The electron burst width is again assumed to be a Gaussian function in time,

$$
I_{1}(t)=\frac{w}{\sqrt{\pi}} e^{-w^{2} t^{2}},
$$

in which $2 \sqrt{\ln 2} / w=p$ is the full width at half maximum of the burst.

2. The neutron source plus detector can be described by a chi-squared function modified by the addition of exponential functions,

$$
I_{2}(t)=A_{0}\left\{\frac{(t+\tau)^{2}}{2 ! \Lambda^{3}} e^{-(t+\tau) / \Lambda}+A_{1}\left[A_{2} e^{-A_{3}\left(t+t_{0}\right)}+A_{4} e^{-A_{5}\left(t+t_{0}\right)}\right]\right\},
$$

where $\tau$ and $\Lambda$ are functions of energy, and $A_{0}$ is the normalization.

3. The time-of-flight channel is again taken to be a square function of time,

$$
I_{3}(t)=\left\{\begin{array}{cl}
1 / c & \text { for }-c / 2<t<c / 2 \\
0 & \text { otherwise }
\end{array},\right.
$$

where the channel width $c$ may be different for different energy-regions.

\subsubsection{Numerical Resolution Function}

Occasionally it may not be possible to fully describe the experimental resolution function with analytic formulae. In that event it may be necessary to give numerical descriptions for each component of the resolution function, in terms of time-of-flight variable $t$, and then to numerically convolute the individual pieces to give the final resolution function. This type of treatment has the virtue of increased flexibility, at the cost of accuracy and speed of calculation. This type of resolution function is available, for example, in the analysis code REFIT; ${ }^{23}$ plans are being made to include this as a fourth option in SAMMY.

\subsubsection{Explicit Normalization and/or Background Functions}

The normalization and background functions available within SAMMY to be added to the theoretical calculations of the cross sections, are described in pages $98 \mathrm{~g}, 122 \mathrm{c}$, and $122 \mathrm{n} .1$ in Revision 4 of SAMMY users' manual. ${ }^{21}$ If $T$ represents the "corrected" theoretical value for cross section, transmission, etc., then $T$ is given by

$$
T(E)=a T_{u}(E)+b(E),
$$

where $T_{u}$ is the uncorrected theoretical value, $a$ is the normalization, and $b(E)$ is the (energydependent) background function. SAMMY offers three methods of specifying backgrounds:

1. Use any or all of the following, but only one of each: 


$$
\begin{array}{ll}
b_{1}(E)=B_{a} & b_{2}(E)=B_{b} / \sqrt{E} \\
b_{3}(E)=B_{c} \times \sqrt{E} & b_{4}(E)=B_{d} \times e^{-B_{f} / \sqrt{E}} .
\end{array}
$$

2. Use as many of the following as needed:

$$
\begin{array}{ll}
b_{1}(E)=A & b_{2}(E)=A e^{-B t} \\
b_{3}(E)=A t^{B} & b_{4}(E)=e^{A+B t+C / \ln (t)},
\end{array}
$$

where time $t$ is derived from the energy using Eq. (32) for fixed value of $\ell$.

3. The user can provide a point-wise description of the background. Note that this method has been available from the beginning, but has not been used extensively; therefore, this option comes with no guarantees.

\subsubsection{Corrections Due to Finite Size of Sample}

Because a physical sample contains a large number of nuclei (on the order of Avogadro's number), it is necessary to make corrections to the calculated theoretical cross sections to include effects resulting from the finite size of the sample.

\subsubsection{Total Cross Section}

For high-resolution experiments in the resolved-resonance region, the easiest measurement (total cross section) is also the easiest to correct for finite size. What is actually measured is not the total cross section per se, but instead the transmission, which is defined as the ratio of the number of neutrons reaching the detector when the sample is in the beam, to the number of neutrons reaching the detector when the sample is not there (sample-in/sample-out). Effectively, a transmission experiment determines the size of the "shadow" of the nuclei in the beam of the neutrons; the thicker the sample, the larger the shadow and the smaller the transmission. The relationship between the (Doppler-broadened) total cross section and the transmission is simply

$$
T=e^{-n \sigma_{t}}
$$

where $n$ is the thickness of the sample (given in units of atoms/barn).

\subsubsection{Partial Cross Sections}

For capture, fission, and scattering cross sections, the effects due to the finite size of the sample are not so easily modeled. For simplicity, this discussion will be confined to capture cross sections; extension to other types of cross sections is straightforward.

Figure 6a depicts the situation for a sample which is a single atom thick, a situation which can never be achieved in an actual experiment. Here the (Doppler- and resolution-broadened) theoretical cross section would correspond directly to the measured cross section. In a slightly thicker sample (Fig. 6b), two effects of finite sample size are noted. First, some nuclei lie in the shadow of other nuclei, and thus have no opportunity to contribute to the cross section; this is called the "selfshielding effect," and results in a lowering of the measured value. Second, some nuclei will have 

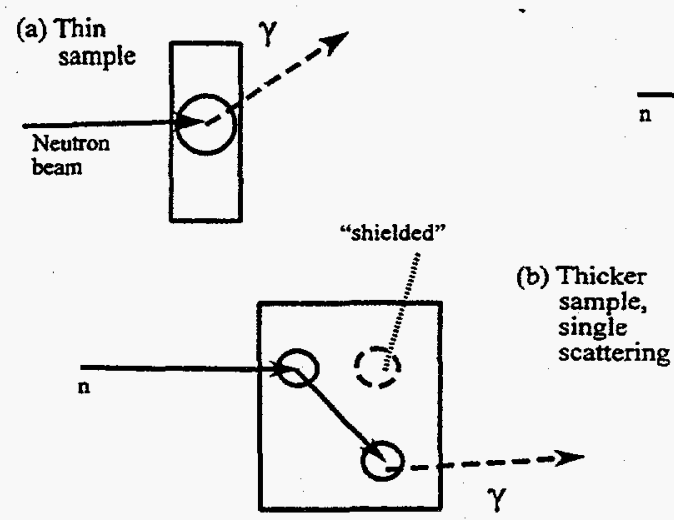

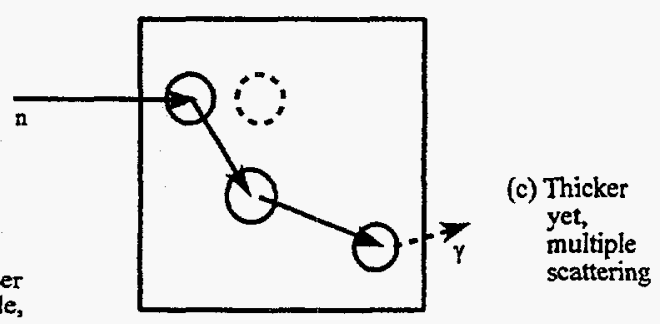

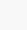

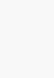

Figure 6: Idealized schematic of a capture experiment using (a) a very thin sample, (b) a slightly thicker sample, and (c) a realistically thick sample.

the opportunity to capture a neutron which has been scattered from another nucleus (and hence is at a lower energy from that of the incident neutron); this is called the "single-scattering correction," and causes an increase in the observed cross section at some energies. In Fig. 6c, the neutron is seen to have scattered two or more times, giving rise to the so-called "multiple-scattering correction."

Each of these effects can be modeled mathematically. Details will be given on pages 560 and following, in Revision 4 of the SAMMY users' guide; complete documentation is also in preparation. ${ }^{25}$ Here we simply quote results.

For self-shielding, the correction is both exact and easy; the self-shielded capture yield may be written in the form

$$
Y_{0}=\left(1-\exp \left(-n \sigma_{t}\right)\right) \sigma_{c} / \sigma_{t}
$$

in which $\sigma_{t}$ is the (Doppler-broadened) total cross section and $\sigma_{c}$ is the (Doppler-broadened) capture cross section.

[Note that there are other ways to normalize the capture yield. SAMMY has three different options, which are explained on pages $56 \mathrm{q}-56 \mathrm{r}$ of revision 4 of the users' manual. ${ }^{21}$ ]

For single-scattering followed by capture, the corrections can be expressed exactly for simplified geometries, in particular for a cylindrical or rectangular sample with the flat face perpendicular to the neutron beam line, as shown in Fig. 7. Both the mathematics and the programming are complicated, because the expressions involve multidimensional integrations. However, if the sample is assumed to be an infinite slab, all but one of the integrations can be performed analytically. The single-scattering correction without edge effects (i.e., infinite-slab approximation) then can be written as an integral over the scattering angle $\theta$ as

$$
\begin{aligned}
Y_{1-} & =2 \pi \int_{0}^{1} d \mu \frac{\sigma_{c}^{\prime}}{\sigma_{t}^{\prime}} \frac{d \sigma}{d \Omega}\left[\frac{1-\exp \left(-n \sigma_{t}\right)}{\sigma_{t}}+\frac{\exp \left(-n \sigma_{t}\right)-\exp \left(-n \sigma_{t}^{\prime} / \mu\right)}{\sigma_{t}-\sigma_{t}^{\prime} / \mu}\right] \\
& +2 \pi \int_{-1}^{0} d \mu \frac{\sigma_{c}^{\prime}}{\sigma_{t}^{\prime}} \frac{d \sigma}{d \Omega}\left[\frac{1-\exp \left(-n \sigma_{t}\right)}{\sigma_{t}}+\frac{\exp \left(-n\left(\sigma_{t}-\sigma_{t}^{\prime} / \mu\right)\right)-1}{\sigma_{t}-\sigma_{t}^{\prime} / \mu}\right],
\end{aligned}
$$


in which primes indicate that quantities are to be evaluated at the scattered energy,

$$
E^{\prime}=E\left[\frac{\cos \theta}{(1+r)}+\sqrt{\frac{1}{(1+1 / r)^{2}}-\frac{\sin ^{2} \theta}{(1+r)^{2}}}\right]^{2},
$$

where $r$ is the ratio of the mass of the nucleus to the mass of the neutron, and $\cos \theta=\mu$.

Even though only the infinite-slab equations are shown here, it is possible within SAMMY to generate the singlescattering correction including edge effects for cylindrical samples. Details are given in the users' manual.

The magnitude of the corrections for self-shielding and single-scattering can be seen in the case of capture in the $1.15-\mathrm{keV}$ resonance of ${ }^{56} \mathrm{Fe}^{24}$ shown in Fig. 8. The "peak" at $1.2 \mathrm{keV}$ is not a resonance, but instead is the result of "scattering into" the resonance at $1.15 \mathrm{keV}$.

For the case of double, triple, etc., scattering (i.e., multiple scattering) followed by capture, corrections to the capture yield can again be expressed explicitly in terms of multidimensional integrals; actual calculations, however, are not possible without making extensive

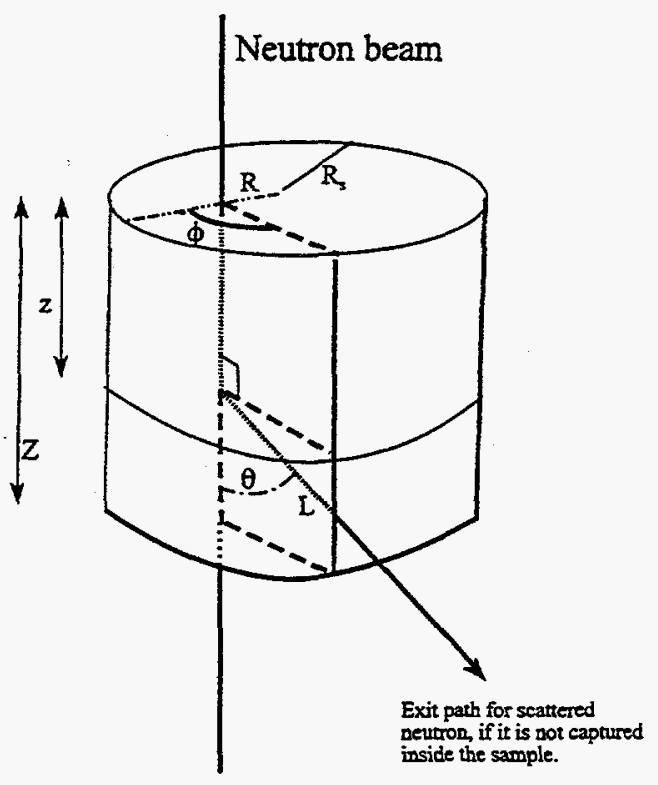
approximations.

The approximations currently used in the treatment of multiple scattering in SAMMY are borrowed from Moxon ${ }^{23}$ but implemented independently: These approximations assume that, after a few scatterings, neutrons are distributed uniformly throughout the sample, and that directions of motion of the neutrons are also distributed uniformly. In other words, the exact (cylindrical) geometry is used, but the memory of where the neutron is and what direction it is moving is eliminated. These assumptions permit effective decoupling of the embedded integrals. The escape probability for a neutron after $k$ scatterings is $e^{-N 0^{k} L}$, where $L$ is the distance $(\mathrm{cm})$ the neutron would travel before leaving the sample, and $N$ is the sample thickness $n$ divided by the physical thickness $Z(\mathrm{~cm})$ of the sample. The average escape probability can then be expressed as

$$
p_{e}\left(E^{(k)}\right)=\frac{1}{n \sigma^{(k)}}\left[\frac{1}{2}-\int_{1}^{\infty} u^{-3} d u e^{-n \sigma^{(k)} u}\right] \text {. }
$$

Thus the approximate correction for capture preceded by $2,3,4, \ldots$ scatterings is given by

$$
\bar{Y}_{2}(E)=N \int d z e^{-N \sigma_{r} z} 2 \pi N \int d \mu_{1} \frac{d \sigma}{d \Omega_{1}} y_{1} \int d q_{1} e^{-N \sigma_{r}^{\prime} q_{1}},
$$



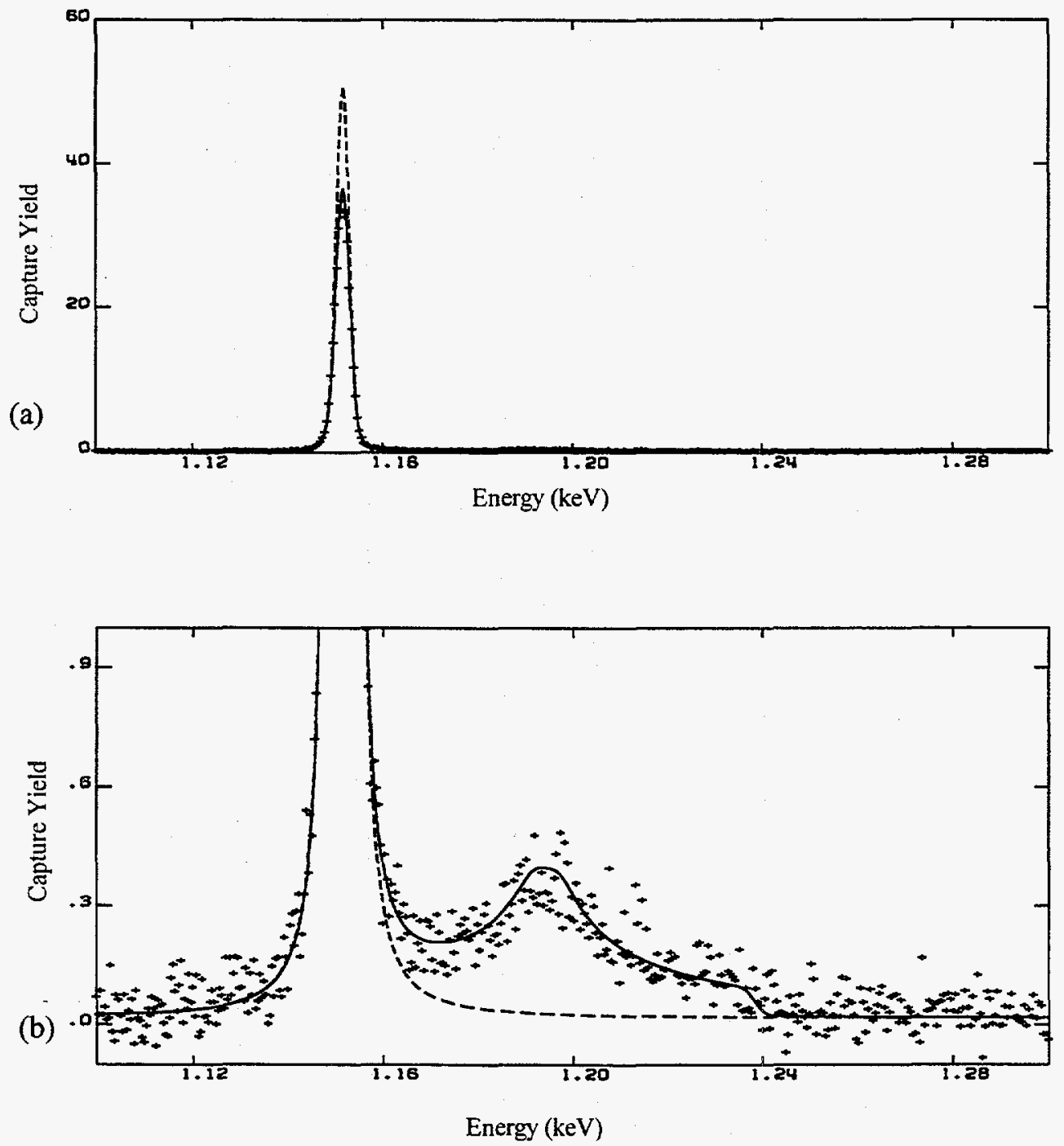

Figure 8: The $1.15-\mathrm{keV}{ }^{56} \mathrm{Fe}$ resonance in natural iron capture data of $\mathrm{R}$. Spencer et al. ${ }^{24}$ Crosses represent the experimental data, the dashed curve is the SAMMY calculation without the self-shielding or single-scattering corrections, and the solid curve includes those corrections. Part (a) shows the complete figure, part (b) gives the lower portion on an expanded scale, to show details of the corrections.

in which the $y_{i}$ are found iteratively from

$$
y_{j-1}=2 \pi \int d \mu_{j} \frac{d \sigma^{(j-1)}}{d \Omega_{j}}\left(\sigma_{c}^{(j)}+y_{j}\right)\left(1-p_{e}\left(E^{(j)}\right)\right)
$$

where the superscript $j$ on the cross section indicates that the cross section is to be evaluated at the energy after $j$ scatterings.

Another option for approximating the effect of double scattering followed by capture (or fission) has been proposed by F. Perey. ${ }^{26}$ In this guise, only those scatterings for which the resulting velocities are aligned in or near the plane of the sample are assumed to be important; others are assumed to escape the sample. This option has not yet been implemented into SAMMY. 


\subsubsection{More than One Type of Nuclide in Sample}

In an actual experimental situation, the sample usually contains more than one type of nuclide. There may be multiple isotopes of the same element, chemical compounds (e.g., oxides), and/or contaminants in the sample. The analysis process must properly account for each particular nuclide, specifying the spin and parity, the mass, and the abundance of each (though the abundance may be treated as a variable whose value is to be determined in the analysis). Appropriate angular momentum algebra for each nuclide must be included, as must the proper kinematics for each nuclide. SAMMY contains provisions to meet all these requirements.

\subsection{Fitting Procedure (Bayes' Method)}

The fitting procedure used in SAMMY is Bayes' method (often called generalized least squares). In the next section one formulation of Bayes' equations will be presented, along with the assumptions underlying its derivation; for additional discussion on this topic, see Ref. 27. Before pursuing details of the methodology, it is useful to discuss why this approach is used rather than the more familiar leastsquares method.

Both Bayes' method and least squares require that "starting" (or a priori) values be provided for all parameters which are to be varied. Implicit in the least-squares equations is the assumption that there is no prior information about the accuracy of the initial parameter values; Bayes' method makes no such assumption. Instead, Bayes' method assumes that prior uncertainties are known for each parameter, and, moreover, that correlations among the parameters are also known. In other words, Bayes' method retains a memory of earlier results. Values and uncertainties from earlier analyses can therefore be used as input to the current analysis; results of sequential analyses of several data sets are identical (with certain restrictions) to results that would be obtained from simultaneous analysis of all the data sets.

Another somewhat peripheral, but nevertheless important, advantage of Bayes' method over least squares is the treatment of irrelevant parameters. Least-squares techniques generally expend considerable time searching for the "best fit" value for parameters which are essentially irrelevant to the problem at hand. (A simple example of this would be a search for the value of the neutron width of a $p$-wave resonance far outside the energy range for the experimental data.) With Bayes' method, this inefficient use of resources does not occur; the irrelevant parameter is treated in a realistic fashion: neither the value nor the uncertainty associated with that parameter is significantly changed.

Logistically, when prior uncertainties and correlations are not known for some parameters, there may be modest problems associated with the use of Bayes' method, since computers do not readily recognize infinities. What is done in practice in SAMMY is to assume large uncertainties and zero correlations for such parameters; generally this works well. The evaluator has the option of modifying the initial values and uncertainties, to test the effects (if any) of such assumptions.

\subsubsection{Derivation of Bayes' Equations}

The derivation of Bayes' equations begins with Bayes' theorem, ${ }^{28}$ which can be written as

$$
p(P \mid D B) \propto p(P \mid B) p(D \mid P B),
$$


Bayes' equations can be derived from Bayes' theorem using three basic assumptions: (1) The prior pdf is a joint normal distribution. (2) The likelihood function is a joint normal distribution. (3) The true value (of the theoretical description for the data) is a linear function of the parameters. A derivation of Bayes' equations is presented in the SAMMY manual; ${ }^{3}$ here we merely state one form of the results:

In one incarnation (denoted the " $\mathrm{M}+\mathrm{W}$ " version in SAMMY), Bayes' equations take the form

$$
\begin{aligned}
P^{\prime}-P & =\left(M^{-1}+W\right)^{-1} Y \\
\left(M^{\prime}\right)^{-1} & =M^{-1}+W \\
Y & =G^{t} V^{-1}(D-T) \\
W & =G^{t} V^{-1} G,
\end{aligned}
$$

in which $P$ represents the initial (a priori) values of parameters, and $P^{\prime}$ represents the final, $M$ represents the initial value of the covariance matrix for the parameters and $M^{\prime}$ represents the final, $D$ represents the values for the experimental data, $V$ is the associated data covariance matrix, $T$ is the theoretical calculation, and $G$ represents the partial derivatives of $T$ with respect to $P$. ( $G$ is sometimes referred to as the "sensitivity matrix.") In the form of Eq. (61), the relationship to the familiar least-squares method is apparent: Take the limit as the diagonal elements of $M$ become infinite (i.e., assume no prior knowledge of the values of parameters), so that the inverse of $M$ is zero. In this case, Eq. (61) reduces to the usual least-squares description.

This one is not the only possible incarnation of Bayes' equations; SAMMY has three different versions, each useful in different situations. In one version (the ' $N+V$ "), the solution of Bayes' equations requires inversion of a dense matrix whose dimension is the number of data points, so this version is useful only when there are few data. A second version (the " $I+Q$ ") is useful primarily when the data covariance matrix is diagonal. The code will automatically choose which version to use in a particular situation, though the user has the option to override that choice if $s /$ he desires.

Mathematically the three formulations of Bayes' equations are equivalent, though results obtained by the various versions may differ slightly due to computer round off and/or nonlinearity considerations.

\subsubsection{Covariance Matrices}

In the previous sections we referred to two different types of covariance matrices, one associated with the data and the other with the parameters. In following sections we shall examine both types in some detail; first we consider why it is important to properly treat covariance information.

\subsubsection{The Importance of Covariances}

In reactor applications, it is often necessary to calculate integral quantities (e.g., reaction rates or $k_{e f f}$ ) of the form

$$
\int_{E \min }^{E_{\max }} \sigma(E) \varphi(E) d E,
$$

where $\varphi(E)$ can be flux or any other function, $\sigma(E)$ is the cross section as calculated from resonance 
parameters, and Emin to Emax can be a large energy range (perhaps 0 to $\infty$ ). Uncertainties on these integral quantities are also needed (that is, we need to know $k_{\text {eff }}$, and we need to know how well we know $k_{e f f}$ ). The calculation of uncertainties on integral quantities requires knowledge of the uncertainties on the quantities within the integrand, and also knowledge of the covariances relating those uncertainties.

Consider first a simplistic example, for which the cross section is a straight line:

$$
\sigma(E)=a E+b \text {. }
$$

Suppose a "data fitting program" has found values, uncertainties, and correlation for $a$ and $b$; assume the value for $a$ is $A$ and the uncertainty $\Delta A$, the value and uncertainty for $b$ are $B \pm \Delta \mathrm{B}$, and the correlation coefficient for the pair is $C$ (where the range on $C$ is -1 to +1 ). Then suppose the integral quantity of interest is

$$
\begin{aligned}
y & =\frac{1}{E \operatorname{Emax}-E \min } \int_{E \max }^{E \max } \sigma(E) d E=\frac{1}{E m a x-E \min } \int_{E \min }^{E \max }(a E+b) d E \\
& =\frac{1}{E \max -E \min }\left(\frac{a}{2}\left(E \max x^{2}-E_{\min ^{2}}\right)+b(E \max -E \min )\right)=a \bar{E}+b,
\end{aligned}
$$

where $\bar{E}$ is the midpoint of the range. The expected value of $y$ is $Y=(A \bar{E}+B)$. The uncertainty on $Y$ is found by taking small increments, squaring, and taking expectation values:

$$
\begin{aligned}
\delta y=\delta a \bar{E}+\delta b \quad\left\langle(\delta y)^{2}\right\rangle & =\left\langle(\delta a \bar{E}+\delta b)^{2}\right\rangle \\
& =\left\langle(\delta a)^{2}\right\rangle \bar{E}^{2}+2\langle\delta a \delta b\rangle \bar{E}+\left\langle(\delta b)^{2}\right\rangle .
\end{aligned}
$$

Rewriting this gives

$$
\Delta^{2} Y=\bar{E}^{2} \Delta^{2} A+2 \bar{E} C \Delta A \Delta B+\Delta^{2} B .
$$

In the usual approximation, the correlation coefficient $C$ is set to zero. Assume that $A=100 \pm 10$, $B=40 \pm 5, E \min =0.5$, and $E \max =1.5$ (which give $\vec{E}=1.0$ ). Then the value for $y$ is $Y=(1)(100)$ $+(40)=140$ with $\Delta^{2} Y=(1)^{2}(10)^{2}+(5)^{2}+2(1) C(10)(5)=125+100 C$. Note that $C=0$ implies $\Delta Y=11.18, C=1$ implies $\Delta Y=15.00$, and $C=-1$ implies $\Delta Y=5.00$. In other words, the uncertainty $\Delta Y$ in this example can vary from $11 \%$ to $4 \%$ of the value for $Y$, depending on the value of the correlation coefficient.

A more realistic example is shown in Fig. 9, which contains artificial fission cross-section data for a uranium-like sample with, however, only one resonance. The "true" values for the resonance parameters are $\mathrm{E}_{0}=3.0300 \mathrm{eV}, \Gamma_{\gamma}=0.0402 \mathrm{eV}, \Gamma_{n}=0.0000195 \mathrm{eV}, \Gamma_{f 1}=-0.0342 \mathrm{eV}$, and $\Gamma_{f 2}=$ $0.0688 \mathrm{eV}$. A SAMMY "fit" to this "data" provided the following values and uncertainties for these parameters: $\mathrm{E}_{0}=3.03038 \pm 0.00006, \Gamma_{\gamma}=0.051323 \pm 0.01952, \Gamma_{n}=0.0000218 \pm 0.0000044, \Gamma_{f 1}$ $=-0.036825 \pm 0.01720$, and $\Gamma_{f 2}=0.056754 \pm 0.02115$. The correlation matrix (times 100) for the "fitted" parameters is found in Table 2.

Table 2: The output parameter correlation matrix (times 100) found from fitting the artificial data shown in Fig. 9.

\begin{tabular}{|lrrrrrr}
\hline$E_{0}$ & 100 & & & & \\
$\Gamma_{\gamma}$ & 0 & 100 & & & \\
$\Gamma_{n}$ & & 0 & 100 & 100 & & \\
$\Gamma_{n}$ & & 0 & -31 & -31 & 100 & \\
$\Gamma_{f 2}$ & 0 & -66 & -66 & -51 & 100 \\
\hline
\end{tabular}




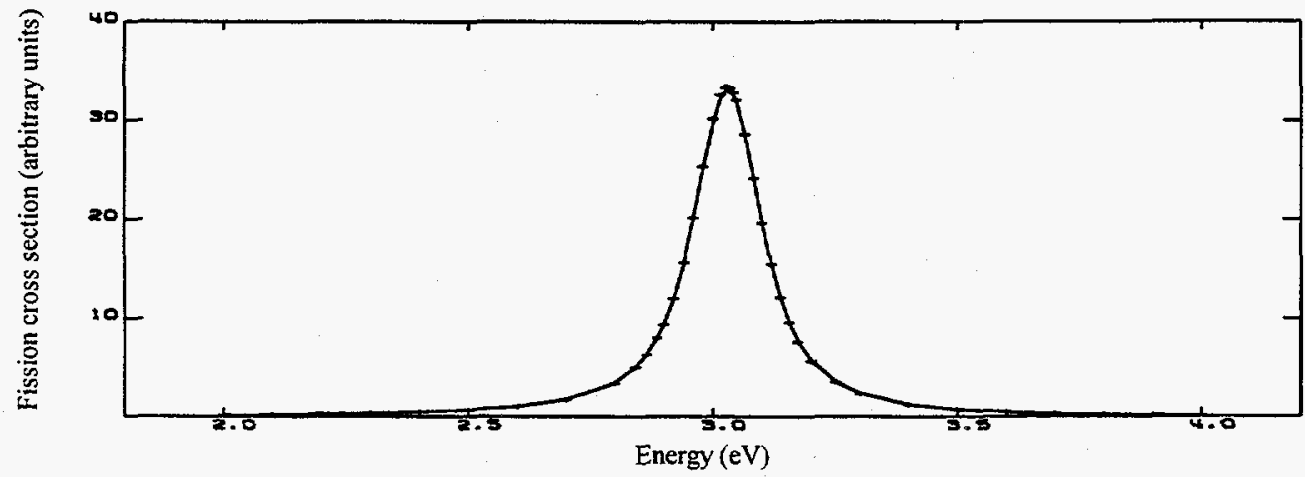

Figure 9: Artificial, uranium-like fission "experimental data" (crosses) and SAMMY fit (solid curve), to iliustrate the use of covariance matrices in calculating group cross-section uncertainties.

Using the "fitted" values for the resonance parameters and uncertainties, the group cross section (averaged from 2 to $4 \mathrm{eV}$ ) can be calculated via SAMMY. When the full covariance matrix is used, the resulting group average is $4.028 \pm 0.026$ barns. When the correct uncertainties are used, but the correlation matrix is assumed to be diagonal, the result is $4.028 \pm 1.061$ barns. Thus the uncertainty on the group average increases from less than $1 \%$ to $26 \%$ when the covariance matrix is not used properly. Similar effects have been observed in real situations.

\subsubsection{Data Covariances}

Obtaining resonance parameters from measured data is a two-step process: the first is data reduction; the second is data analysis. Data reduction is the process of converting from "counts per timechannel" to "cross section as a function of energy"; data analysis is the process of parameterizing the cross section in terms of R-matrix theory. Generally the experimentalist who made the measurement takes care of step 1, the evaluator/analyst takes care of step 2. However, the two processes are not nearly as independent as the definition would suggest; the data covariance matrix provides the linkage between the two steps in the process.

Experimentalists define two types of uncertainties: statistical and systematic. The statistical uncertainty is due to the intrinsic uncertainty in the measurement of the raw data; generally this is governed by Poisson statistics, for which $N$ counts have an uncertainty of $\sqrt{N}$. Systematic uncertainties are due to uncertainties in the measurement of the parameters for the data-reduction process. This process includes such operations as corrections for detector dead time (usually assumed to be exact), normalization by total run time, conversion from time to energy scale, subtraction of backgrounds, dividing sample-in by sample-out counts (if transmission), etc. It is the data-reduction process that leads to off-diagonal data covariance matrix elements. (The raw data are mutually independent; hence the associated covariance matrix is diagonal.)

Over the years, various methodologies have been developed to treat the data covariances. In this section, five such methodologies will be considered in some detail: (1) the "conventional" approach; (2) a better approach; (3) the "usual" approach; (4) the hybrid approach; and (5) the implicit data covariance method. 
A simple example, using artificial data (intended to resemble ${ }^{241} \mathrm{Am}$ ), will be used to illustrate each of these methods. Assume the "raw data" are related to the experimental fission cross section as $r_{i}=\left(1030 \times \sigma_{i}+4970\right.$ barns $)$; this relationship is, of course, not known precisely by the experimentalist, nor by the analyst. Assume the uncertainty on $r_{i}$ is given by

$$
\Delta r_{i}=\sqrt{r_{i}} \text {. }
$$

Assume the experimenter measured the normalization (erroneously) as $950 \pm 60$, and the background (also erroneously) as $1000 \pm 1000$. The question to be answered is the following: Which of the five methods can give the correct resonance parameters and hence the correct cross section?

Method 1, the conventional approach. This is the approach that most analysts would use if they could; it is generally regarded as correct.

The reduced data are functions both of the raw data and of the data-reduction parameters; this function can be written, in general, as

$$
d_{i}=D\left(r_{i}, r_{\tilde{i}}, q_{1}, q_{2}, q_{3}, \ldots\right),
$$

where the $q_{\mathrm{i}}$ represent data-reduction parameters. (For a detailed example, see Ref. 29.) In our simple example, only normalization and background are included, so the reduced data has the form

$$
d_{i}=a r_{i}+b
$$

Although the covariance matrix for the raw data is diagonal, even for this simple case, the covariance matrix for the reduced data is not diagonal, but takes the form

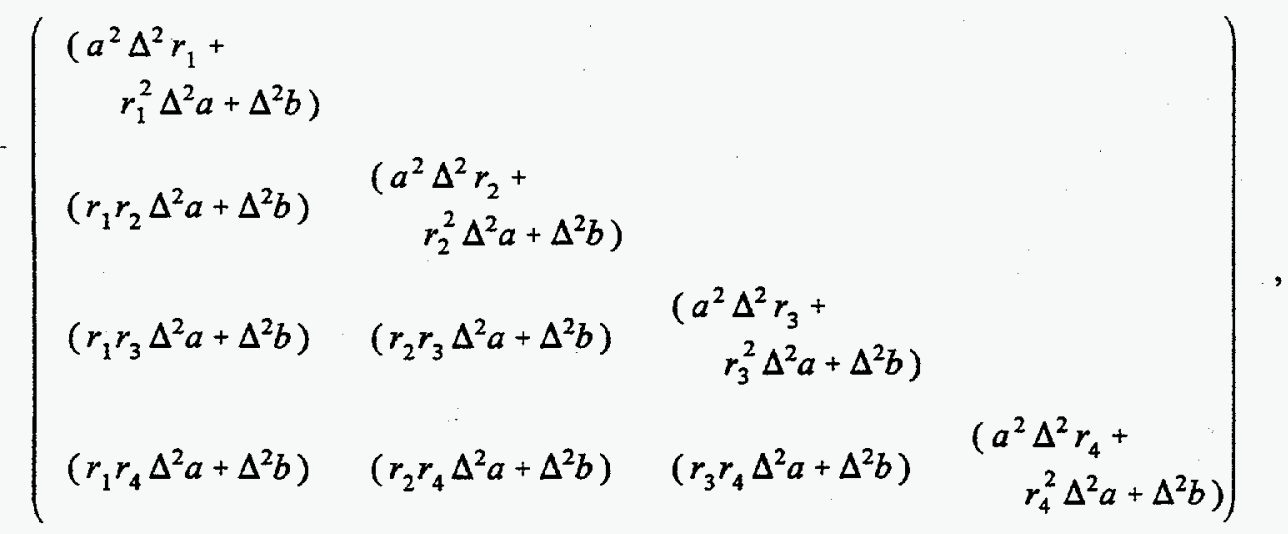

in which only the lower triangular portion of the symmetric matrix is shown.

[In the general case, both the data-reduction procedure and the resulting data covariance matrix are more complex:

$$
\begin{aligned}
d_{i} & =D\left(r_{i}, r_{i}, q_{1}, q_{2}, q_{3}, \ldots\right) ; \\
\delta d_{i} & =\frac{\partial D_{i}}{\partial r_{i}} \delta r_{i}+\frac{\partial D_{i}}{\partial r_{i}} \delta r_{\tilde{i}}+\sum_{k} \frac{\partial D_{i}}{\partial q_{k}} \delta q_{k} ; \text { and } \\
V_{i j} & =\left\langle\delta d_{i} \delta d_{j}\right\rangle=\delta_{i j}\left[\left(\frac{\partial D_{i}}{\partial r_{i}}\right)^{2} \Delta^{2} r_{i}+\left(\frac{\partial D_{i}}{\partial r_{i}}\right)^{2} \Delta^{2} r_{\tilde{i}}\right]+\sum_{k} \sum_{l} \frac{\partial D_{i}}{\partial q_{k}} \frac{\partial D_{j}}{\partial q_{l}}\left\langle\delta q_{k} \delta q_{l}\right\rangle .
\end{aligned}
$$


The computer code ALEX ${ }^{30}$ was designed to facilitate generating $V_{i j}$. In Ref. 29 ALEX was applied to the case of natural nickel data, for which there were 30 data-reduction parameters and 60,000 data points. The lower-triangular portion of the resulting covariance matrix contains $1,800,030,000$ elements, too many to be of practical use.]

"Prior" values for model parameters are found in the literature, or estimated by examining the data. The prior parameter covariance matrix is also estimated. Note that conventional least-squares implicitly assumes that prior uncertainties are infinite and correlations are zero. Bayes' method requires explicit numbers: When uncertainties are known, they should be used; correlations should also be included. When nothing is known, the "non-informative prior" (i.e., infinite uncertainty) should be used. In SAMMY the default non-informative prior is set to $10 \%$ of the parameter value for most variables; generally this is effectively infinite.

Once prior values have been determined, SAMMY (or another analysis code) is then used to analyze the data to find parameter values that give the "best fit" to the data. The output includes both parameter values and the parameter covariance matrix.

Figure 10 depicts the results of this analysis for our simple example. At first glance, the fitted (solid) curve does not appear to match the data; closer inspection, however, reveals that the shape of the curve is correct, only the magnitude and position of the curve are wrong. In Fig. 11, the fitted curve has been modified by the "true" values of the normalization and background parameters, and is seen to lie directly on top of the data points. Hence the values for the resonance parameters given by this method should be correct. (The resonance parameter values determined by each of the five methods are listed in Table 3.)

Even though it provides correct results, there are several disadvantages to this method: For actual measurements, it is not often possible to calculate, store, and manipulate the complete covariance matrix; there are simply too many numbers. Using this method, one obtains no information regarding the values of the data-reduction parameters, so modifying the fitted curve to correspond to the "experimental" data (as in Fig. 11) is generally not possible; hence the fit will often appear, on first inspection, to be poor.

Method 2, a better approach, is to fit the raw data directly. The experimentalist does not reduce the data, but does determine exactly what would be needed to convert to cross sections. Likewise, the reduced data covariance matrix is not generated. In the analysis process, prior values for the model parameters and for the data-reduction parameters must be determined, as must the prior parameter covariance matrix, including uncertainties and covariances for data-reduction parameters. The analysis code then calculates the cross section and converts to whatever function was actually measured, in essence performing the data-reduction process in reverse. These calculated values for the raw data are then fitted to the experimental data; in the process the analyst learns the "best-fit" values not only for the resonance parameters, but also for the data-reduction parameters.

Results from this analysis for our simple example are shown in Fig. 12. Note that the fitted curve (solid line) now agrees well with the data; again, values for resonance parameters found with this method should be correct.

The disadvantage of this method is that it is generally impractical: Analysis codes must include all the data-reduction procedures. In addition, experimentalists are unlikely to publish "counts per channel" rather than "cross sections," especially since they would also have to publish any and all information regarding the data-reduction process. 
Table 3: Comparison of resonance parameter values found using the methods described in the text. There are actually five parameters for each resonance (energy $E_{\lambda}$, widths $\Gamma_{\lambda \gamma}, \Gamma_{\lambda n}, \Gamma_{\lambda f l}$, and $\Gamma_{\lambda f}$, but only those combinations relevant to fission cross sections for isolated resonances are listed here $\left(E_{\lambda}, \Gamma_{\lambda}=\Gamma_{\lambda \gamma}+\Gamma_{\lambda n}+\Gamma_{\lambda j}+\Gamma_{\lambda f 2}\right.$, and $\left.\Gamma_{\lambda f}=\Gamma_{\lambda \eta 1}+\Gamma_{\lambda j 2}\right)$.

\begin{tabular}{|lccccccc|}
\hline Method & a priori value & 1 & 2 & 3 & $3^{\prime}$ & 4 & 5 \\
\hline $\mathrm{E}_{1}(\mathrm{eV})$ & 0.3000 & 0.3071 & 0.3061 & 0.3073 & 0.3069 & 0.3061 & 0.3061 \\
$\Gamma_{1}(\mathrm{meV})$ & 47.39 & 50.69 & 50.63 & 60.46 & 76.54 & 50.65 & 51.40 \\
$\Gamma_{1}(\mathrm{meV})$ & 0.3200 & 0.3391 & 0.3890 & 0.4231 & 0.5075 & 0.3879 & 0.4112 \\
$\mathrm{E}_{2}(\mathrm{eV})$ & 0.5900 & 0.5783 & 0.5769 & 0.5767 & 0.5784 & 0.5769 & 0.5767 \\
$\Gamma_{2}(\mathrm{meV})$ & 48.15 & 56.81 & 52.96 & 68.91 & 116.86 & 52.98 & 51.88 \\
$\Gamma_{2 f}(\mathrm{meV})$ & 0.6000 & 0.4855 & 0.5276 & 0.6491 & 1.0314 & 0.5265 & 0.5383 \\
$\mathrm{E}_{3}(\mathrm{eV})$ & 1.270 & 1.270 & 1.270 & 1.269 & 1.269 & 1.270 & 1.270 \\
$\Gamma_{3}(\mathrm{meV})$ & 47.69 & 47.09 & 46.89 & 59.56 & 62.21 & 46.89 & 46.84 \\
$\Gamma_{3 f}(\mathrm{meV})$ & 0.8001 & 0.5682 & 0.6935 & 0.7994 & 0.8070 & 0.6908 & 0.7257 \\
\hline
\end{tabular}

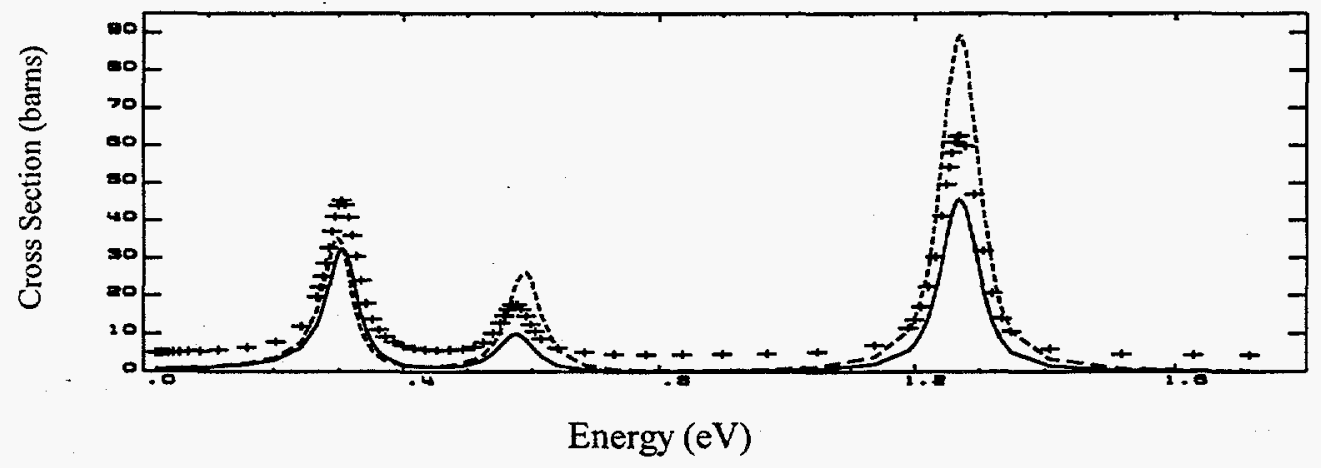

Figure 10: The fit to the "fission cross section for ${ }^{241} \mathrm{Am}$," using Method 1 (fit reduced data using covariance matrix). The crosses represent the reduced data, the dashed curve is calculated from the a priori parameter values, and the solid curve shows the SAMMY fit.

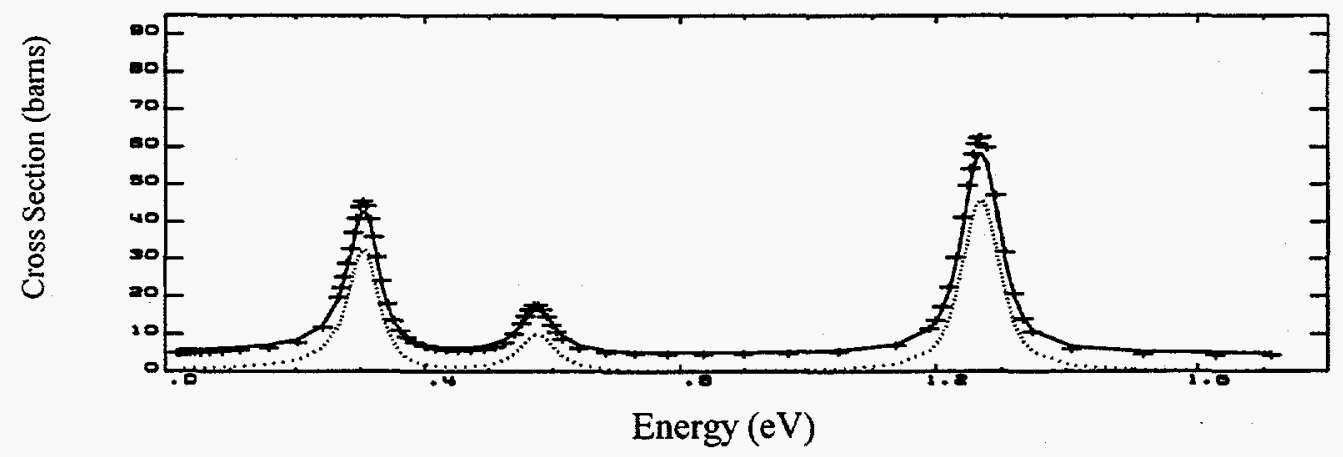

Figure 11: The same fit shown in Fig. 10, with the dotted curve representing the SAMMY fit (as shown in solid curve in Fig. 10), and the solid curve showing the SAMMY fit adjusted by the "true" values of the data-reduction parameters $(1.17 \times$ dotted +4.91$)$. 


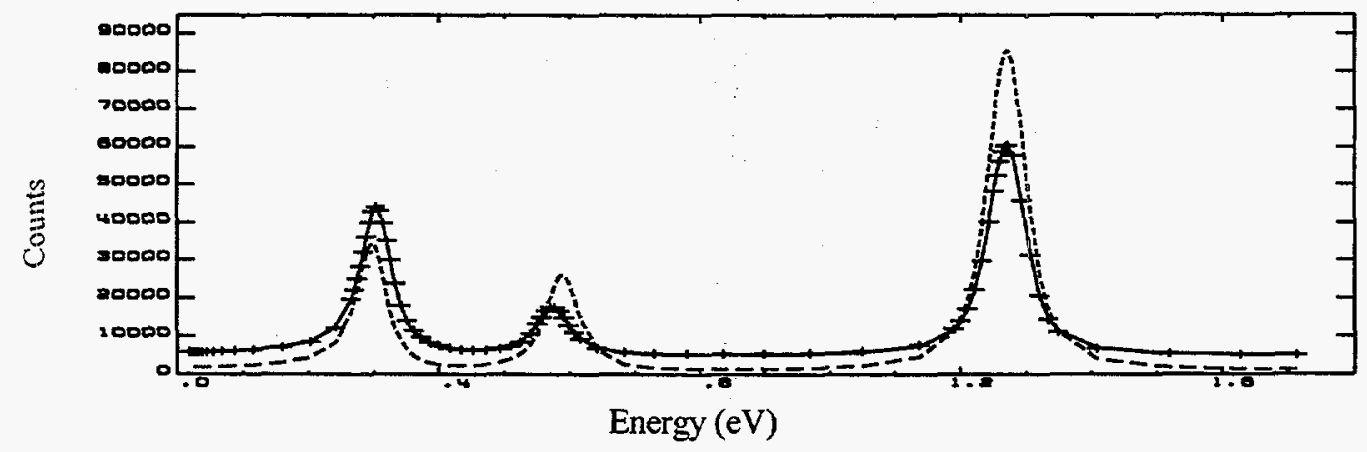

Figure 12: Fission cross section for ${ }^{241}$ Am using Method 2, "a better approach" (fit raw data using datareduction operations in reverse). The crosses represent the raw data, the dashed curve was calculated with the a priori parameter values, and the solid curve shows the SAMMY fit to the raw data.

Method 3 is termed the "usual" approach, because this method is the one in most common use (even though, as will be seen, it can give erroneous results). This method is identical to the "conventional" approach, except that the off-diagonal elements of the covariance matrix for the reduced data are summarily dropped (i.e., set to zero).

Results from the example analysis with this method are shown in Fig. 13. Although the fit is excellent for the peaks, the fit is bad in the valleys. Hence the resonance parameter values are not likely to be correct, as can be seen in Table 3. Obviously a fit this poor would not be acceptable to an evaluator; however, this problem may occur when the poor quality of fit is not so obvious, as when the data in the valleys are noisy. Evaluators are therefore urged to use this method only with great caution, since it can distort results and/or hide inconsistencies.

A variant of this method is to use only statistical uncertainties, rather than including on-diagonal systematic uncertainties as well. This variant is wrong and should never be used. Results from the analysis of our simple example using this variant are included as Method 3' in Table 3; the resonance parameters obtained with this variant are quite different from those obtained with other methods.

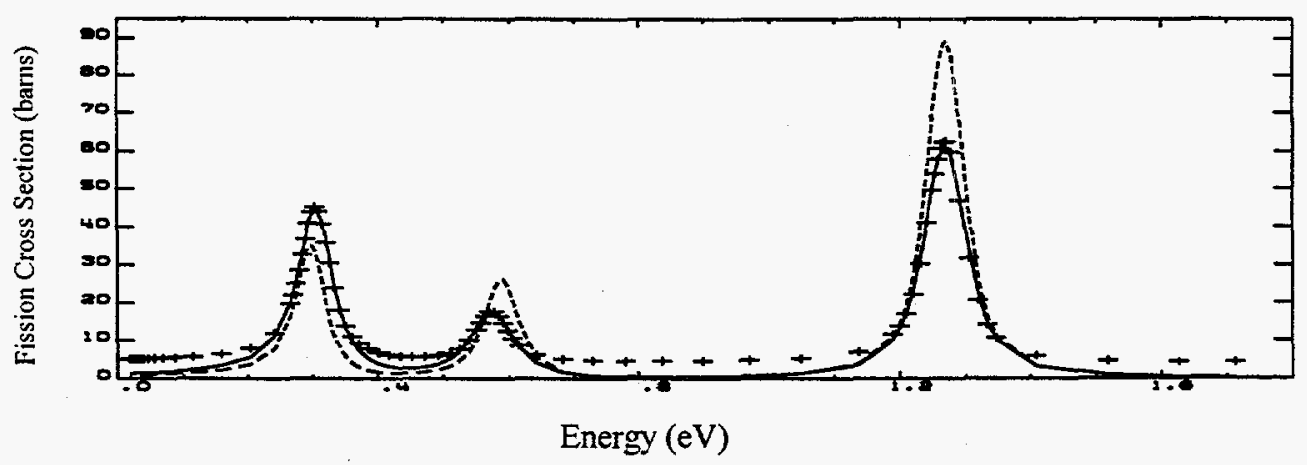

Figure 13: Fission cross section for ${ }^{241}$ Am using Method 3, "the usual approach" (fit reduced data using diagonal portion of covariance matrix). The crosses represent the reduced data, the dashed curve is calculated from the $a$ priori parameter values, and the solid curve is the SAMMY fit. Note that the shape of the fitted curve is wrong. 


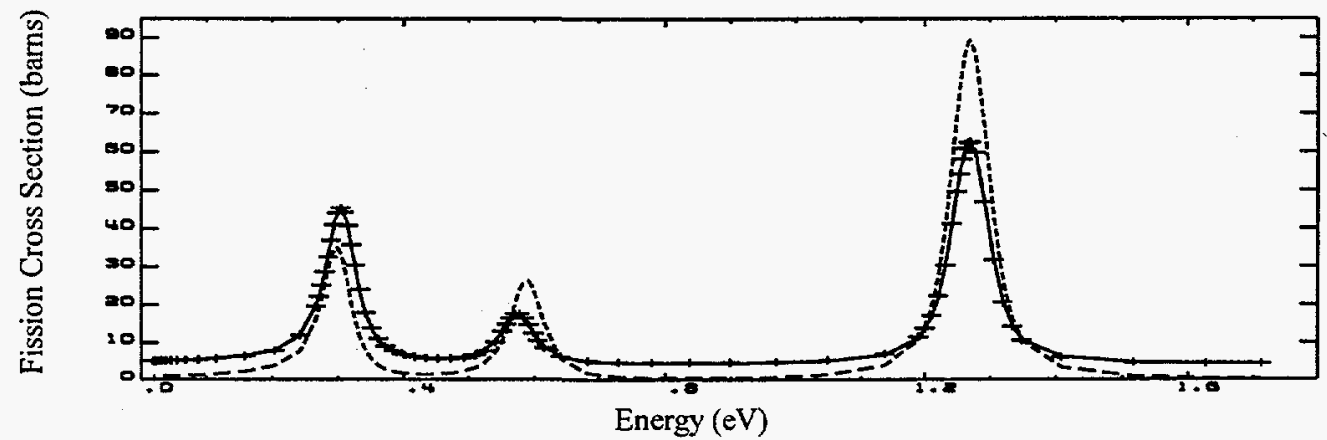

Figure 14: Fission cross section for ${ }^{241} \mathrm{Am}$ using Method 4 (fit reduced data but include pseudo data-reduction operations). Crosses represent the reduced data, the dashed curve was calculated with a priori parameter values, and the solid curve is the SAMMY fit.

Method 4 is the hybrid method, in which the data-reduction process proceeds as though the datareduction parameters were known exactly (i.e., had zero uncertainty); hence the covariance matrix for the reduced data is diagonal and contains only statistical uncertainties. However, for the analysis process, pseudo data reduction parameters (pdrp) are included. These pdrp duplicate the actions of the data reduction parameters, but the a priori values of the pdrp are chosen to have no effect on the value of the calculated cross sections; in our simple example, the initial value of the pseudo normalization is 1 , and of the pseudo background is 0 . The a priori uncertainties for the pdrp should reflect the measured or estimated uncertainties of the actual data-reduction parameters; here, the normalization uncertainty would be 0.050 and the background uncertainty would be 1.00 .

Results of this analysis are shown in Fig. 14. Note that the fitted curve falls on the data, so the resonance parameter values are expected to be correct. With this method, correct values of the pdrp are also found: the normalization is $0.905 \pm 0.041$, and the background is $4.929 \pm 0.056$. Because these values are very different from the input values of 1 and 0 , it is clear that a mistake was made during the original data-reduction process.

Method 5, the implicit data covariance method, is a new addition to SAMMY which is expected to be widely used in the future. In this approach, the analyst provides SAMMY with information about the data reduction parameters, but does not need to explicitly generate the covariance matrix; SAMMY will effectively perform that calculation. In general, the data covariance matrix has the form

$$
V^{i j}=v^{i} \delta_{i j}+\sum_{k} X_{k}^{i} w_{k} X_{k}^{j}
$$

in which $V$ is the data covariance matrix, $v$ represents the statistical uncertainties, $X$ is the sensitivity matrix (partial derivative of data with respect to data-reduction parameters), and $w$ is the covariance matrix for the data-reduction parameters. The inverse of $V$ can be written as

$$
V^{-1}=\left(v+X w X^{t}\right)^{-1}=v^{-1}-v^{-1} X\left(w^{-1}+X^{t} v^{-1} X\right)^{-1} X^{t} v^{-1}
$$

where the dimension of $V$ is thousands $\times$ thousands, of $v$ is thousands $\times$ thousands (but diagonal), of $X$ is thousands $\times$ tens, and of $w$ is tens $\times$ tens (and usually diagonal). This equation is used for the inverse of $V$ as needed in Bayes' equations, thus eliminating the need for inversion of large matrices.

Analysis of our example with this method gives virtually identical results to those found via Method 1. Fig. 15 shows a variation of this approach, using a combination of Methods 4 and 5: Results from the analysis of Method 4 are used to adjust the reduced data (i.e., to correct the improper 


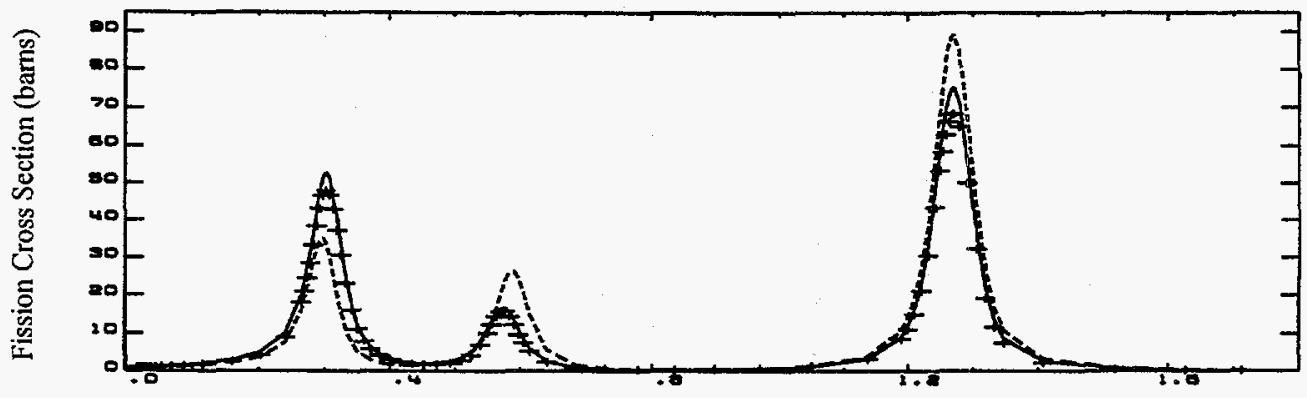

Energy (eV)

Figure 15: Fission cross section for ${ }^{241}$ Am using Method 5, implicit data covariance (fit adjusted reduced data). Crosses represent the adjusted reduced data, the dashed curve was calculated with the a priori parameter values, and the solid curve is the SAMMY fit to the adjusted reduced data.

original data reduction); the adjusted reduced data then are analyzed using Method 5. The virtue of this treatment is that the adjusted data correspond more directly to the true cross section, a great advantage when analyzing and comparing data from several experiments.

\subsubsection{Parameter Covariance Matrices}

The usual, expected output from any analysis of neutron-induced cross-section data in the resolved resonance region is a set of resonance parameters which can then be used to reproduce the "true" cross sections. Another important output from any such analysis is the covariance matrix for those parameters; the covariance matrix provides both the uncertainty for each parameter (square root of the diagonal element of the covariance matrix) and a description of the relationships (correlations) among the various parameters. Both kinds of information must be utilized when calculating uncertainties on derived quantities. For example, in the calculation of multigroup cross sections (which are then used in additional calculations of relevance to nuclear criticality safety concerns), vastly different uncertainties on the final calculations can result if the parameter covariance matrix is not utilized properly.

It is possible, using SAMMY, to generate multigroup cross sections including the associated covariance matrix, starting from the resonance parameters and the covariance matrix associated with those parameters. This capability was used extensively for the first time by Derrien et al. ${ }^{31}$ for ${ }^{235} \mathrm{U}$; results will not be repeated here. The procedure used is to first fit the differential data (and the integral data; see Sect. 4 of this paper), generating values and covariances for the resonance parameters, then use those parameter values to calculate point-wise cross sections that are integrated numerically to give the multigroup cross sections. Included in the procedure is the propagation of uncertainties, yielding the covariance matrix.

\section{Integral Quantities in SAMMY}

Until very recently, the evaluation of differential data and the application of differential cross section information to integral data have proceeded essentially independently. Available differential data (transmission, capture, absorption, fission, eta, etc.) were fitted, using an analysis code such as 
SAMMY, to give appropriate resonance parameters and uncertainties. Those resonance parameters were then used for "data testing" [i.e., for calculating integral quantities (Maxwellian averages, resonance integrals, average integrals, $k_{\text {eff }}$ etc.) which were then compared with "standard" values]. Based on the agreement or disagreement of calculated values to standard values, the resonance parameters were deemed acceptable or unacceptable. When unacceptable, there were few options: often the differential evaluation would need to be reconsidered (a time-consuming and expensive process); sometimes (in extreme cases) the resonance parameters would be modified to give agreement with the integral data without regard to the differential data.

A better methodology ${ }^{32}$ has now been incorporated into SAMMY. In this scheme, certain integral data are included directly within the analysis process, so that the result of the analysis is a set of resonance parameters (plus covariance matrix) which provides a good fit to both differential and integral data. Subsequent data testing on other integral quantities is far more likely to result in agreement of calculation with standards than was the case with the old methodology.

Listed below are the integral quantities that have been implemented into SAMMY. The code will calculate values for all of these; the fitting process includes only those for which the analyst supplies experimental or standard data and uncertainties.

1. Thermal cross section, with subscript $x$ referring to fission, absorption, or capture cross section:

$$
\sigma_{0 x}=\sigma_{x}\left(E_{0}\right) \quad \text { for } E_{0}=0.0253 \mathrm{eV} \text {. }
$$

2. Maxwellian average at thermal energy:

$$
\bar{\sigma}_{x}=\int_{E_{1}}^{E_{2}} \sigma_{x}(E) \frac{E}{E_{0}} e^{-E / E_{0}} d E / \int_{E_{1}}^{E_{2}} \frac{E}{E_{0}} e^{-E / E_{0}} d E,
$$

where $E_{1}=10^{-5} \mathrm{eV}$ and $E_{2}=3 \mathrm{eV}$.

3. Westcott's g-factor :

4. Resonance integral:

$$
g_{x}=\frac{2}{\sqrt{\pi}} \frac{\bar{\sigma}_{x}}{\sigma_{0 x}} .
$$

$$
I_{x}=\int_{E_{3}}^{E_{4}} \sigma_{x}(E) \frac{d E}{E}+X_{4 x}
$$

5. Average integral:

$$
\hat{\sigma}_{x}=\int_{E_{5}}^{E_{6}} \sigma_{x}(E) d E /\left(E_{6}-E_{5}\right)
$$

6. Watt spectrum average:

$$
\bar{\sigma}_{W f}=\int_{E_{1}}^{E_{7}} \sigma_{f}(E) \Phi(E) d E / \int_{E_{1}}^{E_{7}} \Phi(E) d E,
$$

with $E_{7}=20 \mathrm{MeV}$, and $\Phi(E)=$ Watt fission spectrum:

$$
\Phi(E)=e^{-E / a} \sinh (\sqrt{b E})=e^{-E / a}\left(e^{\sqrt{b E}}-e^{-\sqrt{b E}}\right) / 2 .
$$


7. $K 1$ (indicative of $k_{e f f}$ for thermal benchmarks):

$$
K 1=v \sigma_{0 f} g_{f}-\sigma_{0 a} g_{a}=\left(v \bar{\sigma}_{f}-\bar{\sigma}_{a}\right) 2 / \sqrt{\pi} \text {. }
$$

8. Alpha:

$$
\alpha=I_{c} / I_{f}
$$

Other integral quantities will be added to SAMMY in the future; in particular, flux-weighted group cross sections will be added. These are defined by

$$
\bar{\sigma}_{g}=\int_{E_{g}}^{E_{g+1}} \sigma(E) \Phi(E) d E / \int_{E_{g}}^{E_{z^{+1}}} \Phi(E) d E,
$$

with several options for $\Phi(E)$. One option has $\Phi(E)$ given numerically on an energy grid appropriate for intermediate spectra; these will be useful in criticality predictability. Another option uses the Bondarenko narrow-resonance weighting scheme,

$$
\Phi(E)=C(E) /\left[\sigma_{0}+\sigma_{t}(E, T)\right],
$$

in which $C(E)$ is a smooth function of energy, $\sigma_{0}$ is known as the dilution, and $\sigma_{t}(E, T)$ is the Doppler-broadened total cross section. Covariances will of course be calculated for the group cross sections.

\section{Higher-Energy Regions}

A modest beginning has been made to extending the capabilities of SAMMY from the resolvedresonance region on up into higher-energy regions. The ultimate goal is to combine the analyses of all energy regions into one consistent whole. In practical terms, this means to match results at boundaries of the regions.

(1) Many SAMMY analyses, whether intended or not, extend into the "pseudo-resolved" region, in which it is possible to use the resolved-resonance scattering theory to reproduce the cross sections, yet the resonance parameters thus obtained are not truly single-resonance parameters. See, for example, Ref. 5 for a discussion of this matter as it relates to the ${ }^{235} U$ analysis.

(2) To treat the unresolved-resonance region, Fröhner's code FITACS ${ }^{33}$ can be used. This code uses Hauser-Feshbach theory with width fluctuations. The adjustable parameters are strength functions, distant-level parameters, average radiation widths (at $\mathrm{E}=0$ ), and average fission widths (at $E=0$ ). The energy dependence of the radiation widths is specified via the giant dipole model, of the fission widths via Hill-Wheeler fission barrier transmission coefficients, and of the mean level spacing for $s$-waves via the Gilbert-Cameron composite formula. Mean spacings for $l>0$ are given via Bethe formula. Moldauer's prescription is used for partial cross sections.

FITACS has been incorporated into SAMMY in a limited fashion: Internal changes were made, to be consistent with SAMMY notation and to use dynamic dimensioning of arrays. The $M+W$ version of Bayes' method has replaced the fitting procedure used in FITACS. Calculation of penetrabilities has been extended to all $l$ values. The new output includes files from which plots can be made. Results are reported in SAMMY.PAR in the same format as is used in the input file (as well as in more legible fashion in SAMMY.LPT). No other significant changes have been made in the FITACS input, method of calculation, or output; changes are planned, however. The primary 
change will be to use the results of the analysis of the resolved-resonance region as input for the analysis of the unresolved region, in order to provide more consistent evaluated cross sections. Other changes, such as generalization of the theory, redesign of input and/or output, etc., will be made as experience indicates their need.

(3) Treatments for the high-energy regions are also being studied. The likely first candidate to be added to SAMMY is the code GENOA, ${ }^{34}$ an optical model with fitting capabilities, which calculates total, shape elastic and reaction cross sections. GENOA was written by Francis Perey and is being revived by Peter Fu, who is also in the process of rejuvenating his code TNG. ${ }^{35}$ TNG has more capabilities for cross section generation (Hauser Feshbach, pre-equilibrium) than does GENOA, but lacks the superstructure for fitting the variables (i.e., does not calculate the necessary partial derivatives). Other codes and other techniques are being considered for the very high-energy region.

\section{Details about the Code}

The code SAMMY was begun about 1978, when the need became apparent for a state-of-the-art analysis code for use with neutron-induced cross section data from the Oak Ridge Electron Linear Accelerator. SAMMY's progenitor was MULTI, ${ }^{19}$ written by George Auchampaugh at Los Alamos National Laboratory. Although many features of MULTI were retained in the early SAMMY, enough major revisions were implemented to warrant the new name. Chief among these revisions was the change from least-squares fitting procedures to Bayes' method.

Initial SAMMY development work was on the DEC PDP1 0 computer; other early work was on the VAX with VMS operating system. Current development work is done on a UNIX-based IBM workstation; the same version (with minor modifications) runs under Linux, on other unix-based computers, and on the DEC alpha with VMS operating system. SAMMY has also been ported (though not by the author) to Windows95 using Lahey FORTRAN.

SAMMY contains approximately 90,000 lines of "standard" FORTRAN, plus a few lines of coding in the $C$ language. The program is actually a collection of about 50 semi-autonomous codes, although that feature is not obvious to the casual user. For most SAMMY runs, the "master program" organizes which sub-codes are to be invoked in what order; the sub-codes pass information among themselves by means of temporary files (named SAM??.DAT).

Extensive documentation exists for the code. The users' manual ${ }^{3,21}$ contains approximately 400 pages of information; in addition, numerous documents ${ }^{14,20,22,25,27,31,32,36}$ have been written on individual aspects of the program.

SAMMY is currently in use around the United States (ORELA, KAPL, LANL, TUNL, ...) and around the world (Belgium, Brazil, Bulgaria, France, Japan, ...). The code is available from the Radiation Safety Information Computational Center. ${ }^{37}$ Members of OECD can obtain SAMMY from the OECD Nuclear Energy Agency. ${ }^{38}$

\subsection{Input to SAMMY}

Three kinds of files are needed for (almost) every SAMMY run:

(1) The INPut file defines the particular run to be made. This file begins with a title card, which is followed by miscellaneous information such as the minimum and maximum energies for this run, 
Table 4: Sample NPut file, for ${ }^{235} \mathrm{U}$ fission data.

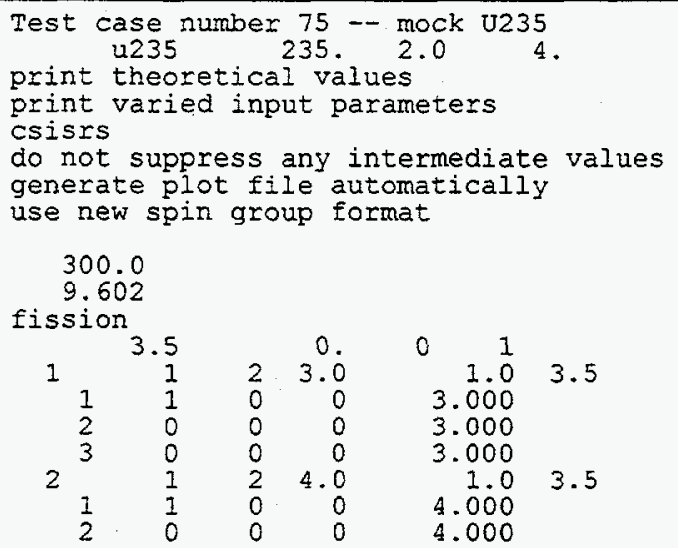

and the density for the auxiliary grid if defaults are to be overridden. Several lines of alphanumeric control information specify details for this run, such as input and output requirements, whether to solve Bayes' equations, which type of R-matrix to use, which Doppler broadening function is to be used, which resolution function, whether to produce group averages, etc. Doppler- and resolutionbroadening parameters are listed, and the data type is specified. Masses and quantum numbers are given for the sample nuclides and for the resonances. Size information for multiple-scattering corrections, and angles for detectors if the data are angular distributions, are also specified in the NPut file. Table 4 contains an example of an INPut file.

(2) The PARameter file gives values for all the resonance parameters, including the energy, the capture width, the neutron width, and any other relevant widths (inelastic, fission, etc.) for each resonance. In addition to resonance parameters, values are given for any other varied parameters, and a priori uncertainties may be given. A small portion of the ${ }^{235} \mathrm{U}$ PARameter file is shown in Table 5; this is a typical example of a SAMMY PARameter file for fissile nuclei. Table 6 shows a very different PARameter file, this one containing resonances for three barium isotopes plus considerable other information (data-reduction parameters, isotopic masses, etc.).

Table 5: First portion of a PARameter file for ${ }^{235} \mathrm{U}$. The final column (labeled "\#") specifies which spin group (set of quantum numbers) applies to this resonance.

\begin{tabular}{|llllllllll|}
\hline Energy in $\mathrm{eV}$ & $\Gamma_{\gamma}$ in $\mathrm{meV}$ & $\Gamma_{\mathrm{n}}$ in meV & $\Gamma_{\mathrm{f}}$ in $\mathrm{meV}$ & $\Gamma_{\mathrm{f}}$ in $\mathrm{meV}$ & flags & & & $\#$ \\
\hline$-2.0383 \mathrm{E}+03$ & $3.3792 \mathrm{E}+01$ & $1.9703 \mathrm{E}+01-4.6652 \mathrm{E}+01-1.0088 \mathrm{E}+02$ & 0 & 0 & 0 & 0 & 0 & 1 \\
$-1.8121 \mathrm{E}+03$ & $3.7445 \mathrm{E}+01$ & $8.5740 \mathrm{E}-01$ & $7.3617 \mathrm{E}+02-7.4187 \mathrm{E}+02$ & 0 & 0 & 0 & 0 & 0 & 1 \\
$-1.5862 \mathrm{E}+03$ & $3.4439 \mathrm{E}+01$ & $8.2845 \mathrm{E}+00$ & $1.5365 \mathrm{E}+02-9.9186 \mathrm{E}+01$ & 0 & 0 & 0 & 0 & 0 & 1 \\
$-1.3575 \mathrm{E}+03$ & $3.8506 \mathrm{E}+01$ & $5.0787 \mathrm{E}+01-1.6914 \mathrm{E}+02-3.8622 \mathrm{E}+02$ & 0 & 0 & 0 & 0 & 0 & 1 \\
$-1.1321 \mathrm{E}+03$ & $3.9794 \mathrm{E}+01$ & $1.7144 \mathrm{E}+03$ & $4.7701 \mathrm{E}+02-4.6937 \mathrm{E}+02$ & 0 & 0 & 0 & 0 & 0 & 2 \\
$-7.2239 \mathrm{E}+02$ & $3.6122 \mathrm{E}+01$ & $2.5036 \mathrm{E}+03$ & $7.7494 \mathrm{E}+02-8.3009 \mathrm{E}+02$ & 0 & 0 & 0 & 0 & 0 & 2 \\
$-5.1588 \mathrm{E}+02$ & $3.8030 \mathrm{E}+01$ & $2.9884 \mathrm{E}+03-8.1285 \mathrm{E}+02-8.1805 \mathrm{E}+02$ & 0 & 0 & 0 & 0 & 0 & 1 \\
$-3.2436 \mathrm{E}+02$ & $3.8934 \mathrm{E}+01$ & $1.5196 \mathrm{E}+02$ & $7.6083 \mathrm{E}+02-7.7511 \mathrm{E}+02$ & 0 & 0 & 0 & 0 & 0 & 2 \\
$-7.4766 \mathrm{E}+01$ & $5.2085 \mathrm{E}+01$ & $3.8375 \mathrm{E}+02-8.6440 \mathrm{E}+02-7.8652 \mathrm{E}+02$ & 0 & 0 & 0 & 0 & 0 & 1 \\
$-3.4928 \mathrm{E}+00$ & $3.7807 \mathrm{E}+01$ & $8.5218 \mathrm{E}-05-6.8909 \mathrm{E}+00$ & $1.2977 \mathrm{E}+01$ & 0 & 0 & 0 & 0 & 0 & 1 \\
$-2.6993 \mathrm{E}+00$ & $3.7012 \mathrm{E}+01$ & $5.6422 \mathrm{E}+00$ & $2.3596 \mathrm{E}+02-1.0107 \mathrm{E}+02$ & 0 & 0 & 0 & 0 & 0 & 2
\end{tabular}


Table 5 , contd.

$\begin{array}{lllllllllll}-1.5027 \mathrm{E}+00 & 3.7237 \mathrm{E}+01 & 8.7360 \mathrm{E}-05-7.2798 \mathrm{E}+00 & 1.2287 \mathrm{E}+01 & 0 & 0 & 0 & 0 & 0 & 1 \\ -9.4548 \mathrm{E}-01 & 3.9404 \mathrm{E}+01 & 5.7502 \mathrm{E}-02 & 1.3397 \mathrm{E}+03-2.6963 \mathrm{E}+00 & 0 & 0 & 0 & 0 & 0 & 1 \\ -1.6052 \mathrm{E}-01 & 1.5678 \mathrm{E}+01 & 1.1942 \mathrm{E}-02 & 1.4586 \mathrm{E}+01-8.8782 \mathrm{E}+00 & 0 & 0 & 0 & 0 & 0 & 2 \\ 3.65750 \mathrm{E}-05 & 4.0000 \mathrm{E}+01 & 6.4608 \mathrm{E}-08-5.0912 \mathrm{E}-01 & 9.3536 \mathrm{E}-01 & 0 & 0 & 0 & 0 & 0 & 2 \\ 3018678427 & 4.0705 \mathrm{E}+01 & 4.8608 \mathrm{E}-03 & 1.2139 \mathrm{E}+02 & 1.9661 \mathrm{E}-01 & 1 & 1 & 1 & 1 & 1 & 1 \\ 1.132577300 & 3.1770 \mathrm{E}+01 & 1.4131 \mathrm{E}-02 & 9.8006 \mathrm{E}-02 & 1.2898 \mathrm{E}+02 & 1 & 1 & 1 & 1 & 1 & 2 \\ 1.308605790 & 4.5120 \mathrm{E}+01 & 1.9938 \mathrm{E}-04-1.8055 \mathrm{E}-01 & 1.8730 \mathrm{E}+01 & 1 & 1 & 1 & 1 & 1 & 2 \\ 2.037662983 & 3.8027 \mathrm{E}+01 & 9.2671 \mathrm{E}-03-9.8491 \mathrm{E}+00 & 9.3994 \mathrm{E}-01 & 1 & 1 & 1 & 1 & 1 & 1 \\ 2.767624855 & 4.1413 \mathrm{E}+01 & 6.6991 \mathrm{E}-04 & 1.2854 \mathrm{E}+02-1.5081 \mathrm{E}-01 & 1 & 1 & 1 & 1 & 1 & 2 \\ 3.147307158 & 4.4826 \mathrm{E}+01 & 2.4926 \mathrm{E}-02-1.8744 \mathrm{E}+01 & 8.7105 \mathrm{E}+01 & 1 & 1 & 1 & 1 & 1 & 1 \\ 3.614400625 & 3.6904 \mathrm{E}+01 & 4.3883 \mathrm{E}-02-5.1667 \mathrm{E}+01-6.5359 \mathrm{E}+00 & 1 & 1 & 1 & 1 & 1 & 2 \\ 4.850314617 & 3.7288 \mathrm{E}+01 & 5.1995 \mathrm{E}-02-1.3173 \mathrm{E}-01-4.4703 \mathrm{E}+00 & 1 & 1 & 1 & 1 & 1 & 2 \\ 5.440935612 & 4.0000 \mathrm{E}+01 & 2.4099 \mathrm{E}-02-3.0152 \mathrm{E}+02-2.0229 \mathrm{E}+02 & 1 & 1 & 1 & 1 & 1 & 2 \\ 6.171471119 & 4.0000 \mathrm{E}+01 & 6.4725 \mathrm{E}-02-5.6557 \mathrm{E}+01 & 1.5807 \mathrm{E}+02 & 1 & 1 & 1 & 1 & 1 & 1 \\ 6.388704300 & 3.8969 \mathrm{E}+01 & 2.3809 \mathrm{E}-01 & 8.4392 \mathrm{E}-01 & 1.0962 \mathrm{E}+01 & 1 & 1 & 1 & 1 & 1 & 2 \\ 7.080036640 & 3.8348 \mathrm{E}+01 & 1.1299 \mathrm{E}-01 & 3.8625 \mathrm{E}+00-3.0033 \mathrm{E}+01 & 1 & 1 & 1 & 1 & 1 & 2 \\ 7.638670444 & 4.0000 \mathrm{E}+01 & 3.0291 \mathrm{E}-03 & 5.8333 \mathrm{E}+01 & 1.0432 \mathrm{E}+02 & 1 & 1 & 1 & 1 & 1 & 1\end{array}$

Table 6: Some of the resonance parameters and data-reduction parameters from a PARameter file for barium isotopes.

\begin{tabular}{|c|c|c|c|c|c|c|c|c|c|c|}
\hline Energy in $\mathrm{eV}$ & $\Gamma_{\gamma}$ in $\mathrm{meV}$ & $\Gamma_{n}$ in meV & & & flags & & $\#$ & & & \\
\hline $\begin{array}{l}6042.66846 \\
6127.59473 \\
6128.08789 \\
6167.77783 \\
6178.40723 \\
6209.12891 \\
6303.52002 \\
6530.13379 \\
6607.07324 \\
6643.58057 \\
6769.49609 \\
6862.02148\end{array}$ & $\begin{array}{l}8.9000 \mathrm{E}+01 \\
7.3000 \mathrm{E}+01 \\
8.0700 \mathrm{E}+01 \\
1.6030 \mathrm{E}+02 \\
7.3000 \mathrm{E}+01 \\
6.7800 \mathrm{E}+01 \\
7.7000 \mathrm{E}+01 \\
7.3000 \mathrm{E}+01 \\
8.0610 \mathrm{E}+01 \\
1.9600 \mathrm{E}+02 \\
7.2700 \mathrm{E}+01 \\
6.2300 \mathrm{E}+01\end{array}$ & $\begin{array}{l}9.9400 \mathrm{E}+ \\
1 \\
1.1000 \mathrm{E}+ \\
1.1620 \mathrm{E}+ \\
3.9300 \mathrm{E}+ \\
2.9200 \mathrm{E}+ \\
1.3040 \mathrm{E}+ \\
7.5800 \mathrm{E}+ \\
3.5750 \mathrm{E}+ \\
1.6600 \mathrm{E}+ \\
1.9500 \mathrm{E}+ \\
1.6900 \mathrm{E}+ \\
1 \\
1.6000 \mathrm{E}+\end{array}$ & $\begin{array}{l}02 \\
-01 \\
04 \\
02 \\
-01 \\
01 \\
03 \\
03 \\
-01 \\
-02 \\
-01 \\
-02 \\
-01\end{array}$ & & $\left.\begin{array}{ll}0 & 0 \\
0 & 0 \\
0 & 0 \\
0 & 0 \\
0 & 0 \\
0 & 0 \\
0 & 0 \\
0 & 0 \\
0 & 0 \\
0 & 0 \\
0 & 0 \\
0 & 0\end{array}\right\}$ & $\begin{array}{l}0 \\
0 \\
0 \\
0 \\
0 \\
0 \\
0 \\
0 \\
0 \\
0 \\
0 \\
0\end{array}$ & $\begin{array}{r}1 \\
3 \\
12 \\
13 \\
3 \\
2 \\
1 \\
3 \\
2 \\
13 \\
2 \\
2\end{array}$ & $\begin{array}{l}1 \\
3 \\
2 \\
3 \\
3 \\
2 \\
1 \\
3 \\
2 \\
3 \\
2 \\
2\end{array}$ & & \\
\hline \multicolumn{10}{|c|}{ 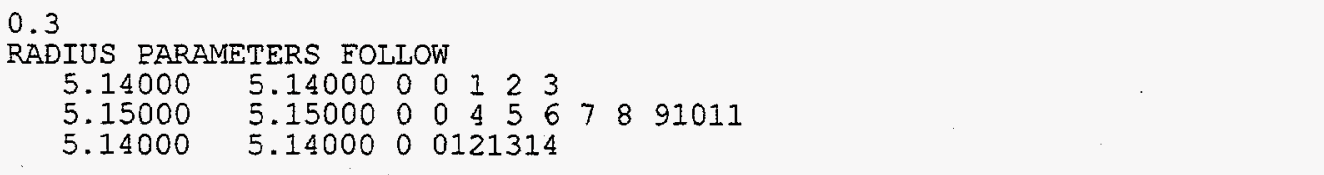 } & \\
\hline \multicolumn{11}{|c|}{ 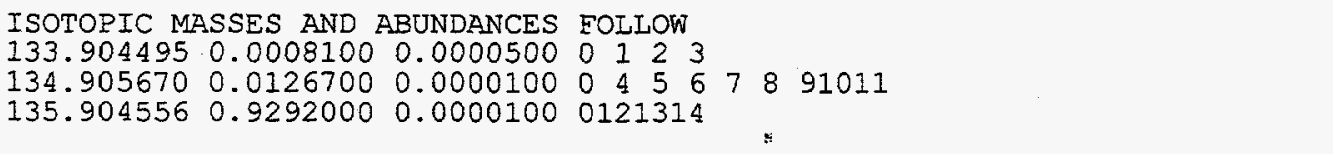 } \\
\hline $\begin{array}{r}\text { BROADENING } \\
5.140000\end{array}$ & $\begin{array}{c}\text { PARAMETERS } \\
300.000\end{array}$ & $\begin{array}{l}\text { FOLLOW } \\
0.002788\end{array}$ & 0.030000 & 0.020000 & 0.000000 & 0 & $0-2$ & & 00 & 0 \\
\hline $\begin{array}{ll}\text { ORRESO lutio } \\
\text { BURST } & 0 \\
\text { WATER } & 0004 \\
\text { WATER } & 0004 \\
\text { IITHI } & 000 \\
\text { LITHI } & \\
\text { CHANN } & 0 \\
\text { CHANN } & 0 \\
\text { CHANN } & 0 \\
\text { CHANN } & 0 \\
\text { CHANN } & 0 \\
\text { CHANN } & 0 \\
\text { CHANN } & 0 \\
\text { CHANN } & 0\end{array}$ & $\begin{array}{r}\text { function } \\
7.000 \\
4.165 \\
1.000 \\
1.000 \\
0.100 \\
27.949 \\
58.712 \\
116.675 \\
224.719 \\
415.621 \\
1089.655 \\
3672.072 \\
6540.650\end{array}$ & $\begin{array}{r}\text { parameter } \\
1.000 \\
-0.089 \\
0.009 \\
0.692 \\
0.000 \\
512.000 \\
256.000 \\
128.000 \\
64.000 \\
32.000 \\
16.000 \\
8.000 \\
6.000\end{array}$ & $\begin{array}{r}\text { follow } \\
0.037 \\
0.004 \\
1.000 \\
0.100 \\
0.100 \\
0.100 \\
0.100 \\
0.100 \\
0.100 \\
0.100 \\
0.100 \\
0.100\end{array}$ & & & & & & & \\
\hline $\begin{array}{l}\text { NORMAl izati } \\
1.0000000 \\
0.1\end{array}$ & $\begin{array}{l}\text { on and "con } \\
1.213 \mathrm{E}-04 \\
1.000 \mathrm{e}-05\end{array}$ & $\begin{array}{l}\text { istant" ba } \\
.385 \\
.04\end{array}$ & $\begin{array}{l}\text { ckground } \\
0.000 \mathrm{E}+00\end{array}$ & $\begin{array}{l}\text { follow } \\
0.000 \mathrm{E}+00\end{array}$ & $0.000 E+00$ & 0 & 10 & & 0 & 0 \\
\hline
\end{tabular}


Table 7: MULTI-style DATa file (relative uncertainties). Note that 105 columns per line are required.

\begin{tabular}{|c|c|c|c|c|c|c|c|c|}
\hline Energy & Cross Section & Unc. & Energy & Cross Section & Une & Energy & Cross Section & Une \\
\hline 167852.60 & 7.5705647 & .05140 & 167561.00 & 7.7268302 & .05088 & 167379.20 & 7.7512475 & .05080 \\
\hline 167306.50 & 7.7813070 & .05070 & 167233.90 & 7.8170076 & .05058 & 167161.40 & 7.8635455 & .05043 \\
\hline 167088.80 & 7.8557944 & .05046 & 267016.40 & 7.9129777 & .05027 & 166944.00 & 7.9459873 & .05017 \\
\hline 166763.10 & 8.0105363 & .04997 & 166474.40 & 8.1585770 & .04951 & 166186.40 & 8.3154668 & .04904 \\
\hline 165899.20 & 8.5065538 & .04849 & 165612.70 & 8.7009951 & .04794 & 165326.90 & 8.9387033 & .04730 \\
\hline
\end{tabular}

(3) The DATa file contains the energies, measured value for the experimental data, and uncertainty on the data. At least four format options are available, of which two are most commonly used: The original, or MULTI-style, format has three data points per line and requires relative uncertainties; format is $3(2 \mathrm{E} 14.8, \mathrm{~F} 7.5)$. The CSISRS format uses one data point per line, absolute uncertainties, in 3F11.8 format. Table 7 shows a few lines of a MULTI-style DATa file, and Table 8 shows data in CSISRS format.

Other files are needed for some SAMMY runs: (4) The COVariance file contains the parameter covariance matrix as generated by a previous run. (5) The DCV (data covariance) file contains the covariance matrix for the experimental data. (6) The AVG (average) file contains energy ranges for averaging (i.e., for calculating the multigroup average cross sections). (7) The NDF file contains information needed in order to produce an output parameter file in the ENDF/B-6 format. (8) The MXW file provides the temperatures at which to evaluate stellar (Maxwellian) averages for use in astrophysics applications. (9) The SSM file holds the edge-effects information for single-scattering corrections to capture or fission yields, as generated by a previous SAMMY run. (10) The NTG file holds experimental values for integral quantities.

Table 8: CSISRS DATa file (absolute uncertainties).

\begin{tabular}{|lll|}
\hline Energy in $\mathrm{eV}$ & Cross Section & Uncertainty \\
\hline 95664.094 & 0.6009411 & 0.60094 \\
95666.750 & 0.9116803 & 0.91168 \\
95669.398 & 1.2838910 & 1.28389 \\
95672.047 & 1.6690609 & 1.66906 \\
95674.695 & 2.0074561 & 2.00746 \\
95676.023 & 2.1419671 & 2.14197 \\
95677.344 & 2.2495730 & 2.24957 \\
95678.008 & 2.2913780 & 2.29138 \\
95678.672 & 2.3249400 & 2.32494 \\
95679.336 & 2.3505211 & 2.35052 \\
95680.000 & 2.3675220 & 2.36752 \\
95680.656 & 2.3764009 & 2.37640 \\
95681.320 & 2.3763959 & 2.37640 \\
95681.984 & 2.3683021 & 2.36830 \\
95682.648 & 2.3518860 & 2.35189 \\
95683.969 & 2.2939861 & 2.29399 \\
95685.297 & 2.2065721 & 2.20657 \\
95687.945 & 1.9487320 & 1.94873 \\
95690.594 & 1.6104290 & 1.61043 \\
95693.250 & 1.2344520 & 1.23445 \\
95695.898 & 0.8692769 & 0.86928 \\
\hline
\end{tabular}




\subsection{Output Files from SAMMY}

(1) Every SAMMY run produces a file called SAMMY.LPT, which, as the name suggests, was originally intended to be printed on a line printer. SAMMY users should learn to routinely examine this file, to be sure that input was properly understood by the code, and to learn details about the run and about the output. Included in this file are, for example, values for varied and fixed parameters (what you actually told SAMMY to use, not what you intended to tell SAMMY to use!), verbatim alphanumeric commands (with notification if a command is unacceptable), input file names, the title for the run (from the INPut file), a chronological listing of modules used during the run, updated parameter values, uncertainties, and correlations (also intermediate values if requested), $\chi^{2}$ values, and error messages (which are often duplicated on-screen or in a log file). No sample of an LPT file is given here, but users are strongly encouraged to become familiar with this file.

(2) The file SAMMY.IO gives only the input and output parameter values, in the same format in which they appear in the LPT file.

(3) A "normal" SAMMY run, in which Bayes' equations are solved in order to find values of the parameters that provide a good fit to the data, will produce at least two other output files, SAMMY.PAR and SAMMY.COV. SAMMY.PAR contains new (updated) values of resonance parameters and other varied parameters, in the same format as the input PARameter file; the file also contains a line indicating that a corresponding covariance file exists. This new PAR file, with or without modification, can be used as input to another SAMMY run.

(4) SAMMY.COV is a binary file containing the covariance matrix for the varied parameters; it also contains "exact" values for all parameters. Both SAMMY.PAR and SAMMY.COV should be renamed at the end of a run, before using them as input to another run.

(5) The file SAMMY.ODF contains the energy grid, the experimental data, the absolute uncertainties for the experimental data, and the initial and final calculated values for cross section. It may also contain uncertainties on the calculated values, and/or a revised energy grid. This file is intended to be used for making plots of data vs theory. The format for this file is either ODF (ORELA Data Format) or "generic" binary, the decision to be made by the system manager, depending on the plotting package used at each facility. SAMMY.ODF should be renamed before beginning another SAMMY run, to avoid losing information.

Other output files will be produced when requested (via commands in the INPut file): (6) File SAMMY.NDF gives resonance parameters in ENDF/B-6 format. (7) SAMMY.PDS contains partial derivatives in ASCII format. (8,9) SAMNDF.INP is the SAMMY-style INPut file, produced when ENDF/B File 2 is used for input instead of SAMMY-style PARameter file; SAMNDF.PAR is the associated SAMMY-style PARameter file. (10) SAMXAC.ODF is a binary file containing the auxiliary grid and unbroadened cross section. (11) SAMMY.SSM is a binary file with edge-effects information for the single-scattering correction for capture or fission yields. (12) SAM??.DAT are temporary files used for communication between the separate modules of the SAMMY program; these files can be deleted at the end of a successful SAMMY run, but should be saved for debugging purposes when a run aborts abnormally. 


\subsection{Debugging}

Novice users of complicated codes like SAMMY are certain to run into difficulties; even experienced users will occasionally encounter problems they cannot solve. This section provides a few guidelines that may help in these situations.

(1) First, do not panic - remember, this happens to everyone!

(2) Is this run really the same as the one that worked last week? Think carefully: exactly what did you change?

(3) Look at the LPT file in detail. Did SAMMY understand all of your instructions? Maybe you had a typographical error. Is SAMMY using the same parameter values that you intended? Maybe you had a typographical error. Look for factors-of-ten. Would additional information help? Perhaps you need to ask SAMMY to print out more initial values, or more intermediate steps. Did SAMMY give you an error message? Look at the very end of the LPT file or LOG file (i.e., at the information which appears on screen if running interactively).

(4) Can you simplify and/or isolate the problem? Try using a smaller energy range, varying fewer parameters, dropping normalization \& background options, doing no broadening. Find the smallest case for which the error occurs, and try to understand what is unique about that case.

(5) If the problem persists, ask for help. Talk to your office mate, or to the most experienced SAMMY user in the neighborhood. Maybe you've just been looking at it for too long, and someone else can see immediately what you can no longer see. It does get easier with practice!

(6) When all else fails, contact the author at nml@ornl.gov. First, send information but not files. Describe the problem in some detail: What version of SAMMY are you using? What features are in use? Did the code bomb or just give wrong answers? In which module did the bomb occur? Does a minor perturbation of this case work properly? When requested, send very small files from the simplest case which has the problem: command file, INPut file, PARameter file, and DATa file. (Send those files as attachments if possible, otherwise as separate e-mail messages.)

NOTE: If you find and fix a bug in your version of SAMMY (either in the code or in the manual), please inform the author about it so that she can fix it in the official version. Also, if you can suggest a more informative error message, please contact the author.

\subsection{SAMMY Features Not Mentioned Elsewhere}

Most of the major features of SAMMY have been discussed elsewhere in this report. For completeness' sake, in this section are listed some of the other features which may be of interest:

It is possible to use ENDF files for input to SAMMY (resonance parameters from File 2 and backgrounds from File 3); however, note that only isotopic files are allowed, not files for natural materials.

An option exists to print the resonance parameters in ENDF format.

Paramagnetic cross sections can be added to total cross sections if needed.

Average (group) cross sections can be calculated either with no corrections on the theoretical values, or with Doppler broadening or other corrections included.

A. first attempt has been made at coding to convert from REFIT ${ }^{23}$ input to SAMMY input (and vice versa). 
Reconstruction of point-wise cross sections from resonance parameters can be accomplished in a variety of ways; one technique for generation of an appropriate energy grid was borrowed from NJOY, another is indigenous to SAMMY.

The auxiliary code SAMAMR is available to rearrange data-reduction variables when doing sequential analyses of unrelated data sets.

File from which to make plots can be generated in one of two ways, producing either an ORELA Data Format file for use with ORNL in-house plotting routines, or a "generic binary file" which can then be plotted using other plotting packages.

Stellar (Maxwellian) averages can be calculated for use in nuclear astrophysics applications.

\subsection{Future Plans for SAMMY}

Although the SAMMY code has been evolving for two decades, it is by no means yet a "finished product." There remain a great many improvements and extensions that will eventually be incorporated into the code. A few of the possible future developments for SAMMY are listed here.

Revision 4 of the users' manual, ${ }^{21}$ plus a new RSICC $^{37}$ release of the FORTRAN (version M2), should become available this calendar year.

Additional testing and improvements are desirable for the multiple-scattering corrections for capture and fission yields.

More work is needed on the unresolved-resonance and the high-energy regions.

Charge-dependent penetrabilities will soon be added to the code, for use with charged-particle final states. This addition may also permit SAMMY to be used to analyze proton-induced reactions in addition to neutron-induced reactions.

Additional Integral Quantities (for intermediate energies) will be added.

An option may be added to use the full R-matrix theory (i.e., with no approximation such as Reich-Moore) to calculate cross sections.

More options may be added for the resolution function.

An option to use time-of-flight rather than energy as the independent variable may be added.

On the wish list, but not funded, is to port SAMMY to WINDOWS95 for ease of use on personal computers. Currently, the code is available in unix and Linux versions, and in VMS. Also on the wish list is the development of a modern graphical user interface for SAMMY.

A major restructuring, to permit truly simultaneous analysis of any and all data sets, is in the planning stages. ${ }^{39}$ This restructuring will use the $\mathrm{M}+\mathrm{W}$ formulation of Bayes' equations (see Sect. 3.3.1 of this paper), in which all data-set-dependence can be isolated into quantities $W$ and $Y$, which are strictly additive with respect to independent data sets. Therefore the contributions to $W$ and $Y$ can be generated separately for each data set, added together, and then used to solve Bayes' Equations once and for all. Current problems involving nonlinearity, ordering of sequential analyses, etc., should thereby be alleviated without increasing the overhead costs associated with storing and manipulating large arrays.

Interfaces are being developed to provide access to the SAMMY parameter covariance matrix for use by processor codes such as $\mathrm{NJOY}^{40}$ or AMPX. ${ }^{41}$ 


\section{A Complete Evaluation}

Finding appropriate resonance parameters for a particular nuclide involves much more than simply gathering data and then running SAMMY once or twice; indeed, doing an evaluation "from scratch" is perhaps more of an art than a science. In this section, guidelines are offered to show how such an evaluation may be accomplished successfully. Classic examples may be found in the work of Claire and Francis Perey on ${ }^{58} \mathrm{Ni}^{42}{ }^{60} \mathrm{Ni}_{,}{ }^{43}$ and ${ }^{56} \mathrm{Fe},{ }^{44}$ as well as in the work of Luiz Leal and Herve Derrien on $\mathrm{Pu}^{45,46}$ and $\mathrm{U}^{5,47}$

Begin by gathering publications, both experimental data and previous analyses (from SAMMY and from other codes). Check the data banks for data files, parameter files, etc. Don't forget thermal values and integral quantities.

Experimental conditions are extremely important. You need to know the resolution for the timeof-flight channels, the flight path length, the sample thickness, everything. See the worksheets in Table 9 for one possibility for keeping track of all these details.

Keep in mind that different data sets may not agree with each other. You will want to make sure that the data sets used in an evaluation are consistent; this may require you to make adjustments (in energy-scale, in normalization and/or backgrounds) for some of data sets. [Don't make any such adjustments early in the evaluation process, but keep in mind that you may need to eventually. $\mathrm{Be}$ sure to keep track of, and report, everything you do!]

Choose one "best" data set to use for your "standard" for energy-scale, location of resonances, etc. Often this would be the transmission experiment with the best resolution.

Choose starting values for resonance parameters. Good sources for information include the data files, ${ }^{37,38}$ Mughabghab, ${ }^{48}$ and published reports and journals. Prepare SAMMY-style INPut and PARameter files, either by typing them yourself or by making a SAMMY run which starts from ENDF resonance parameter files.

Begin with "no-Bayes" runs (i.e., include the line "DO NOT SOLVE BAYES EQUATIONS" in the INPut file) for the energy-differential data. These runs calculate the cross section from the initial parameters, but do not attempt to provide "best values" for any parameters. Study the plot from the no-Bayes runs. Are there systematic energy differences between some experimental data and theory in the energies of the resonances? If so, you may need to adjust the energy scale for this data set.

In the no-Bayes runs, consider whether the Doppler- and resolution broadening are about right. Look at the broadening widths at several energies. (SAMMY reports the values of the widths at EMIN and EMAX in the LPT file; you may wish to look at other energies as well.)

Begin your analyses (the Bayes runs) with the best high-resolution transmission data. (Note: always fit to transmission data, not to total cross section data. Otherwise the blacking-out resonances will give you considerable grief!) Flag the resonance energies and the neutron widths, and search for "best values" for those parameters; use average values (e.g., from Mughabghab) for the capture widths, and estimates for the fission widths. You may wish to increase the number of iterations from the default of 2 , to $\sim 6$, at this initial stage. After the resonance energies and neutron widths are fairly well established, it is time to generate an accurate representation for the external R-matrix. Use the technique described in Sect. 7.1 to do this.

Next consider other types of data. Fission widths should be varied for fission data, and capture widths for capture data. Remember that you may need to adjust (vary) resolution parameters, normalization, and / or background for any given experiment. 
Table 9: Worksheets for keeping track of details of experiments

\begin{tabular}{|l|l|}
\hline & (a) Measurement Descriptions \\
\hline Material: & Natural silicon \\
\hline Data Type: & Transmission but data available as total cross sections \\
\hline Laboratory: & ORNL \\
\hline Experimentalist(s): & D. C. Larson, C. H. Johnson, J. A. Harvey \\
\hline Run Number: & 8090 \\
\hline Date of experiment: & $1 / 7 / 74$ \\
\hline
\end{tabular}

\begin{tabular}{|c|c|}
\hline \multicolumn{2}{|c|}{ (b) Experimental Conditions } \\
\hline Flight Path Length \pm Dispersion (m) & $78.203 \pm 0.022$ \\
\hline Neutron Overlap Filter & $0.60 \mathrm{~cm}$ P.B. $300 \mathrm{mg} / \mathrm{cm}^{2}{ }^{10} \mathrm{~B}$ \\
\hline Permanent Background Samples & Not applicable) \\
\hline Sample for the Determination of Background & Not applicable) \\
\hline Linac Burst Width (ns) & 5.5 \\
\hline Repetition Rate (sec ${ }^{-1}$ ) & 180 \\
\hline Power (kW) & 2 \\
\hline Moderator & (Not applicable) \\
\hline Moderator Thickness & (Not applicable) \\
\hline Detector Type & ${ }^{6}$ Li glass \\
\hline
\end{tabular}

\begin{tabular}{|c|c|}
\hline \multicolumn{2}{|c|}{ (c) Time-of-Flight Analyzer } \\
\hline Energy Range $(\mathrm{keV})$ & Channel Width (ns) \\
\hline $\mathrm{E}<0.007$ & 1024 \\
\hline $0.007<\mathrm{E}<0.091$ & 256 \\
\hline $0.091<\mathrm{E}<0.432$ & 64 \\
\hline $0.432<\mathrm{E}<1.54$ & 32 \\
\hline $1.54<\mathrm{E}<5$ & 16 \\
\hline $5.0<\mathrm{E}<13.9$ & 8 \\
\hline $13.9<\mathrm{E}<\infty$ & 4 \\
\hline
\end{tabular}

\begin{tabular}{|c|c|}
\hline \multicolumn{2}{|c|}{ (d) Sample Characteristics } \\
\hline Weight (grams) & (Not needed) \\
\hline Thickness (atm/barns) & 0.07462 \\
\hline Isotopic Composition & (Natural) \\
\hline Sample Composition & (Natural) \\
\hline Impurities, etc) & $23-\mathrm{cm}$ diameter \\
\hline Size (mm) & $0.75 \mathrm{~cm}$ thick \\
\hline
\end{tabular}


Once you have fairly accurate resolution parameters, normalizations, and backgrounds, for each data set, you can make sequential SAMMY runs through all the experimental data in order to refine the values for the resonance parameters. (Use SAMAMR in between the SAMMY runs as needed.)

Does the order of the data sets matter in the sequential runs? Sometimes it might, since Bayes? method assumes linearity but the real world is not linear.

Are there discrepancies between data sets? Those should become apparent as you make sequential runs; values for parameters may change considerably whenever you use a certain data set. You may need to "throw out" bad data, or renormalize; study the literature on that experiment and see if there is information you have not included properly. Or perhaps there is something the authors neglected to explain. Look carefully at the LPT files as you make the runs. Note especially when values of Chi-squared change radically.

Do you have the correct spin-group assignments for all resonances?

Consider angular-distribution data if they are available: Often these data do not have good enough statistics to be included directly in the analysis, but they can be very helpful in determining the appropriate spin-group assignments (quantum numbers) for resonances.

Look for interference effects in fission channels and inelastic channels; remember that such effects result only from levels of the same spin state. Also, do not confine yourself to a short energy range; interference effects can be long-range. If necessary, have SAMMY calculate contributions from individual spin groups (by $X$ 'ing out all others -- putting an " $\mathrm{X}$ " in Column 5 for those spin groups -- in the INPut file).

When there is no way to tell precisely which spin assignments are correct, use statistical methods (e.g., Delta-3 statistics) or trial-and-error to find assignments that "work."

Have you considered the possibility that there might be doublets (two resonances of different spin groups at essentially the same energy)?

Once the energy-differential data are fitted quite well, it is time to include the integral data. Add this run to your set of sequential SAMMY runs.

Values for all parameters (for real and "dummy" resonances, for radii, for data-reduction parameters) should be reported in the literature, as should the associated covariance matrices. Use program SAMDIST ${ }^{36}$ to calculate statistical properties, which should also be included in the report.

\subsection{Estimating the Contribution of the External R-matrix}

One method of estimating the contribution of the external resonances is to start with the resonances in the energy range of interest (EMIN $=0$ to EMAX), and to shift this sample into the negativeenergy region and again into the region just above the maximum energy for this evaluation. These two samples of shifted resonances are then used to generate their contribution to the various cross sections (scattering, fission, capture, and total) in the energy range 0 to EMAX. The next step is to reduce the number of external levels to a few $(\$ 20)$ fictitious levels, $\$ 10$ in the negative-energy region and $\$ 10$ above EMAX, to represent the above calculated cross sections. The parameters of these (fictitious) levels are obtained by fitting the calculated cross sections using SAMMY.

This procedure has been used in the evaluation of the resonance parameters of ${ }^{239} \mathrm{Pu},{ }^{45}{ }^{241} \mathrm{Pu},{ }^{46}$ ${ }^{233} \mathrm{U},{ }^{47}$ and ${ }^{235} \mathrm{U} .{ }^{5}$ It appears to be a simple but efficient method since it uses real existing resonances 
and consequently leads to a more physically meaningful external resonance parameter set. This procedure, unlike most other methods of determining external R-matrix parameters, preserves the strength function.

When the external resonance contribution is accurately calculated, a constant effective scattering radius can be used for fitting the neutron transmission data over all the neutron energy range of analysis. In order to evaluate the value of the scattering radius, preliminary SAMMY fits of the experimental neutron transmission data can be performed in several selected energy ranges, using as input the real resonances and the fictitious resonances. The results should be nearly equal, with modest fluctuations about an average value.

\section{Concluding Comments}

This paper is intended to serve as an introduction to the theory underlying the analysis of neutroninduced cross-section data in the resolved resonance region. Three main aspects (scattering theory parameterization, treatment of experimental effects, and fitting procedure) of such analyses were

described. Extensions to integral quantities and to higher-energy regions were discussed. An overview of the analysis code SAMMY was presented. Finally, a procedure was outlined for performing a complete and thorough analysis for any given nuclide.

\section{References}

1. F. H. Fröhner, "Applied Theory of Resolved and Unresolved Resonances," Applied Nuclear Theory and Nuclear Model Calculations for Nuclear Technology Applications, eds. M. K. Mehta and J. J. Schmidt (World Scientific, Singapore, 1989).

2. F. H. Fröhner, "Theory of Neutron Resonance Cross Sections for Safety Applications," KFK 5073, Kernforschungszentrum, Karlsruhe (1992).

3. N. M. Larson, Updated Users' Guide for SAMMY: Multilevel R-matrix Fits to Neutron Data Using Bayes' Equations, ORNL/TM-9179/R3, Oak Ridge National Laboratory, Oak Ridge, TN (1996).

4. N. M. Larson, "Cross Section Parameterization in the Resolved Resonance Region," International Symposium on Nuclear Data Evaluation Methodology, ed. Charles L. Dunford, 285-294 (1993).

5. L. C. Leal, H. Derrien, N. M. Larson, and R. Q. Wright, R-Matrix Analysis of ${ }^{235} U$ Neutron Transmission and Cross Sections in the Energy Range 0 to $2.25 \mathrm{keV}$, ORNL/TM-13516, Oak Ridge National Laboratory, Oak Ridge, TN (1997).

6. J. A. Harvey, N. W. Hill, F. G. Perey, G. L. Tweed, and L. C. Leal, Proc. Int. Conf. on Nuclear Data for Science and Technology, Mito, Japan (May 30-June 3, 1988).

7. L. W. Weston and J. H. Todd, Nucl. Sci. Eng. 111, 415 (1992). 
8. G. de Saussure, R. Gwin, L. W. Weston, and R. W. Ingle, Simultaneous Measurements of the Neutron Fission and Capture Cross Section for ${ }^{235} \mathrm{U}$ for incident neutron energy from $0.4 \mathrm{eV}$ to $3 \mathrm{keV}$, ORNL/TM-1804, Oak Ridge National Laboratory, Oak Ridge, TN (1967).

9. A. M. Lane and R. G. Thomas, "R-Matrix Theory of Nuclear Reactions," Reviews of Modern Physics 30, 257-353 (1958).

10. A. Foderaro, The Elements of Neutron Interaction Theory, The MTT Press, Cambridge, MA, and London, England (1971).

11. ENDF-102, Data Formats and Procedures for the Evaluated Nuclear Data File, ENDF-6, written by the Members of the Cross Section Evaluation Working Group, ed. V. McLane, C. L. Dunford, P. F. Rose, BNL-NCS-4495, Brookhaven National Laboratory, Upton, NY, revised 1995.

12. G. M. Hale and D. C. Dodder, "R-Matrix Analyses of Light-Element Reactions for Fusion Applications," Proc. Int. Conf. on Nuclear Cross Sections for Technology, Knoxville 1979, J. L. Fowler and C. H. Johnson, eds., NBS Special Publication 594, 650 (1980).

13. ENDF-201, ENDF/B-VI Summary Documentation, compiled and edited by P. F. Rose, BNLNCS-17541 [ENDF-201], 4th edition, Brookhaven National Laboratory, Upton, NY 11973 (1991).

14. N. M. Larson, M. C. Moxon, L. C. Leal, and H. Derrien, Doppler Broadening Revisited, ORNL/TM-13525, Oak Ridge National Laboratory, Oak Ridge, TN (1998).

15. L. Leal and R. Hwang, personal communication (1985).

16. D.E. Cullen and C.R. Weisbin, Nuc. Sci. and Eng. 60, 199-229 (1976).

17. A. Meister, Calculations on Lattice Vibration Effects in the Doppler Broadening of the $0.18 \mathrm{eV}$ Cd Neutron Resonance Cross Section, personal communication (1994).

18. M. D. Mintz and D. P. Jordan, A 'Progressive' Interpolation Scheme for Hand and Digital Computer Analysis of Tabulated Data, Lawrence Livermore Laboratory Report UCRL-7681 (1964).

19. G. F. Auchampaugh, MULTI, A FORTRAN Code for Least-Squares Shape Fitting of Neutron Cross-Section Data Using the Reich-Moore Multilevel Formalism, LA-5473-MS, Los Alamos National Laboratory, Los Alamos, NM (1974).

20. N. M. Larson, Oak Ridge Realistic Resolution Function for SAMMY, in preparation.

21. N. M. Larson, ORNL/TM-9179/R4, in preparation.

22. N. M. Larson, RPI Resolution Function for SAMMY, in preparation.

23. M. C. Moxon, REFIT2: A Least Squares Fitting Program for Resonance Analysis of Neutron Transmission and Capture Data, NEA-0914/02, July 1989.

24. R. R. Spencer, N. M. Larson, J. H. Todd, and L. W. Weston, "The ORNL Neutron-Capture CrossSection Facility," presented at the International Conference on Nuclear Data for Science and Technology, Gatlinburg, TN, May 9-13, 1994. Proceedings, International Conference on Nuclear Data for Science and Technology, J. K. Dickens, ed., page 99ff.

25. N. M. Larson, Multiple-Scattering Corrections to Capture and Fission Yields, in preparation.

26. F. G. Perey, personal communication (1990). 
27. N. M. Larson, "Covariances as Input to and Output from Resonance Analysis," Proceedings of a Specialists' Meeting on Evaluation and Processing of Covariance Data, ed. M. Wagner (1993).

28. Bayes' theorem is explained in most books on probability theory; one example is E. Parzen, Modern Probability Theory and its Applications," John Wiley \& Sons, Inc., NY, page 119 (1960).

29. D. C. Larson, N. M. Larson, J. A. Harvey, N. W. Hill, and C. H. Johnson, ORNL/TM-8203, Oak Ridge National Laboratory, Oak Ridge, TN, ENDF-333 (October 1983).

30. N. M. Larson, ORNL/TM-8676, ENDF-332 (February 1984). See also "Uncertainty Propagation from Raw Data to Final Results," Proceedings of the International Conference on Nuclear Data for Basic and Applied Science, Santa Fe, New Mexico, May 13-17, 1985, Vol. 2, ed. Phillip G. Young, et al. (Gordon and Breach Science Publishers )1533-1536 (1986).

31. H. Derrien, N. M. Larson, and L. C. Leal, Covariance Matrices for Use in Criticality Safety Predictability Studies, ORNL/TM-13492 (September 1997). Submitted for publication in Nuclear Science and Engineering.

32. N. M. Larson, L. C. Leal, and H. Derrien, Integral Data Analysis for Resonance Parameters Determination, ORNL/TM-13495 (September 1997). Submitted for publication in Nuclear Science and Engineering.

33. F. H. Fröhner, Nucl. Sci. Eng. 103, 119-128 (1989).

34. F. G. Perey, "GENOA NOTICE NO. 1," personal communication, ORNL, Oak Ridge, TN (1968).

35. C. Y. Fu, A Consistent Nuclear Model for Compound and Pre-Compound Reactions with Conservation of Angular Momentum, ORNL/TM-7042 (1980); K. Shibata and C. Y. Fu, Recent Improvements of the TNG Statistical Model Code, ORNL/TM-10093 (1986); C. Y. Fu, Nucl. Sci. Eng. 86, 344 (1984) and 92, 440 (1986) and 100, 61 (1988).

36. L. C. Leal and N. M. Larson, SAMDIST: A Computer Code for Calculating Statistical Distributions for R-Matrix Resonance Parameters, ORNL/TM-13092, Oak Ridge National Laboratory, Oak Ridge, TN (September 1995).

37. Radiation Safety Information Computational Center (RSICC, formerly Radiation Shielding Information Center), P.O. Box 2008, Oak Ridge, TN 37831-6362, USA; see also the web site http://www-rsicc.ornl.gov/rsic.html.

38. OECD Nuclear Energy Agency, Le Seine-St Germain, 12, boulevard des Îles, F-92130 Issy-les-Moulineaux, France; see also the web site http://www.nea.fr/html/dbprog.

39. N. M. Larson, "Efficient Use of Bayes' Equations for Fitting Neutron-Induced Cross Section and Integral Data," accepted for presentation at the International Conference of the Physics of Nuclear Science and Technology, to be held in October of 1998 on Long Island, NY.

40. R. E. MacFarlane, D. W. Muir, and R. M. Boicourt, The NJOY Nuclear Data Processing System, Volume II: The NJOY, RECONR, BROADR, HEATR, and THERMR Modules, LA-9303-M, Vol. II (ENDF-324) (May 1982). Also R. E. MacFarlane, Introducing NJOY 89, LA-UR 89-2057, Los Alamos National Laboratory, Los Alamos, NM (1989). 
41. N. M. Greene, W. E. Ford III, L. M. Petrie, and J. W. Arwood, AMPX-77: A Modular Code System for Generating Coupled Multigroup Neutron-Gamma Cross-Section Libraries from $E N D F / B-I V$ and/or ENDF/B-V, ORNL/CSD/TM-283, Oak Ridge National Laboratory, Oak Ridge, TN (1992).

42. C. M. Perey, J. A. Harvey, Richard L. Macklin, F. G> Perey, and R. R> Winters, "Resonance parameters of ${ }^{60} \mathrm{Ni}+\mathrm{n}$ from measurements of transmission and capture yields from 1 to 450 keV," Phys. Rev. C 27, 2556 (1983).

43. C. M. Perey, F. G. Perey, J. A. Harvey, N. W. Hill, N. M. Larson, R. L. Macklin, and D. C. Larson, ${ }^{~}{ }^{88} \mathrm{Ni}+\mathrm{n}$ Transmission, Differential Elastic Scattering and Capture Measurements and Analysis Up to 813 keV," Phys. Rev. C 47, 1143-1169 (1993).

44. C. M. Perey, F. G. Perey, J. A. Harvey, N. W. Hill, and N. M. Larson, " ${ }^{\circ 56} \mathrm{Fe}$ and ${ }^{60} \mathrm{Ni}$ Resonance Parameters," Proceedings of the International Conference on Nuclear Data for Science and Technology, 13-17 May 1991, Juelich, Germany, ed. S. M. Qaim, Springer-Verlag Berlin Heidelberg, 41 (1992).

45. H. Derrien, J. Nucl. Sci. Technol. 30(9), 845 (1993).

46. H. Derrien and G. De Saussure, "R Matrix Analysis of the ${ }^{241} \mathrm{Pu}$ Neutron Cross Sections in the Thermal to 300-eV Energy Range," Nucl. Sci. Eng. 106, 434 (1990).

47. H. Derrien,"R-Matrix Analysis of Neutron Effective Total Cross Section, Fission Cross Section and Capture Cross Section of ${ }^{233} \mathrm{U}$ in the Energy Range from Thermal to $150 \mathrm{eV}$," J. Nucl. Sci. Technol., 31(5), 379 (1994).

48. S. F. Mughabghab and D. I. Garber, Neutron Cross Sections, BNL-325, Brookhaven National Laboratory, Third Edition (1973). 
ORNL/M-6576

\section{INTERNAL DISTRIBUTION}

$\begin{aligned} \text { 1. } & \text { B. L. Broadhead } \\ \text { 2. } & \text { H. Derrien } \\ \text { 3. } & \text { F. C. Difilippo } \\ \text { 4. } & \text { M. E. Dunn } \\ \text { 5. } & \text { C. Y. Fu } \\ 6 . & \text { N. M. Greene } \\ 7 . & \text { K. Guber } \\ \text { 8. } & \text { J. A. Harvey } \\ 9 . & \text { C. M. Hopper } \\ \text { 10. } & \text { D. T. Ingersoll } \\ \text { 11. } & \text { P. E. Koehler } \\ \text { 12. } & \text { M. A. Kuliasha } \\ \text { 13. } & \text { D. C. Larson } \\ \text { 14-18. } & \text { N. M. Larson }\end{aligned}$
19. L. C. Leal
20. C. V. Parks
21. R. W. Roussin
22. R. O. Sayer
23. C. H. Shappert
24. M. S. Smith
25. R. R. Spencer
26. R. M. Westfall
27. J. E. White
28. R. Q. Wright
29. RSICC

30-31. Laboratory Records for submission to OSTI

32. Laboratory Records, ORNL-RC

33. Central Research Library

\section{EXTERNAL DISTRIBUTION}

34. P. Blaise, DER/SPRC/LEPH, Batiment 230, Centre d'Etudes de CADARACHE, 13108 Saint Paullez-Durance, France

35. R. Block, Gaerttner LINAC Laboratory, Department of Environmental and Energy Engineering, Rensselaer Polytechnic Institute, Troy, NY 12180-3590

36. O. Bouland, DER/SPRC/LEPH, Batiment 230, Centre d'Etudes de CADARACHE, 13108 Saint Paul-lez-Durance, France

37. A. Brusegan, Central Bureau for Nuclear Measurements, Steenweg op Retie, 2240 Geel, Belgium

38. David Buchan P41 E1, Pickering Nuclear Generating Station, Ontario Hydro, 1675 Montgomery Park Rd., Pickering, Ontario CANADA L1V 2R5

39. J. Burke, Gaerttner LINAC Laboratory, Department of Environmental and Energy Engineering, Rensselaer Polytechnic Institute, Troy, NY 12180-3590

40. D. Cabrilla, U.S. Department of Energy, EM-66, Clover Leaf, Room 1199, 19901 Germantown Road, Germantown, MD 20874-1290

41. D. E. Carlson, Reactor and Plant System Branch, Division of System Research, Office of Nuclear Regulatory Research, U.S. Nuclear Regulatory Commission, MS T-10 G6, RM T-10, I7, Washington, DC 20555-0001

42. M. Chadwick, T-2, MS B243, Los Alamos National Laboratory, P. O. Box 1663, Los Alamos, NM 87545

43. F. Corvi, Central Bureau for Nuclear Measurements, Steenweg op Retie, 2240 Geel, Belgium

44. D. E. Cullen, MS L-298, Lawrence Livermore National Laboratory, P. O. Box 808, Livermore, CA 94550

45. Y. Danon, Physics Department, NRCN, P. O Box 9001, Beer-Sheva 84190, Israel

46. R. L. Dintaman, U.S. Department of Energy, DP-13, Washington, DC 20585

47. C. Dunford, Bldg 197D, National Nuclear Data Center, Brookhaven National Laboratory, Upton, NY 11973 
48. J. R. Felty, U.S. Department of Energy, DP-311, Washington DC 20585

49. P. Finck, Argonne National Laboratory, Reactor Analysis Division, Bldg 208, Argonne, IL 60439

50. E. Fort, DER/SPRC/LEPH, Batiment 230, Centre d'Etudes de CADARACHE, 13108 Saint Paullez-Durance, France

51. N. Francis, Gaerttner LINAC Laboratory, Department of Environmental and Energy Engineering, Rensselaer Polytechnic Institute, Troy, NY 12180-3590

52. C. M. Frankle, NIS-6, MS J562, Los Alamos National Laboratory, Los Alamos, NM 87545

53. S. C. Frankle, X-CI, MS F663, Los Alamos National Laboratory, Los Alamos, NM 87545

54. F. Froehner, Kernforschungszentrum Karlsruhe, Institut fur Neutronenphysik und Reacktortechnik, Postfach 336 40, D-76021 Karlsruhe, Germany

55. W. Furman, Frank Laboratory of Neutron Physics, JINR, Dubna, Russia

56. S. Ganesan, Head, Nuclear Data Section, Indira Gandhi Centra for Atomic Research, Kalpakkam 603 102, Tamilnadu, India

57. H. Gruppelaar, Netherlands Energy Research Foundation ECN, Westerduinweg 3, P. O. Box 1, NL 1755 ZG Petten, Netherlands

58. F. Gunsing, Centre D'Etudes De Saclay, F-Saclay - 91191 GIF-SUR-YVETTE Cedex, France

59. G. M. Hale, T-2, MS B243, Los Alamos National Laboratory, P. O. Box 1663, Los Alamos, NM 87545

60. A. Hasagawa, Nuclear Data Center, Japan Atomic Energy Research Institute, Tokai-mura, Nakagun, Ibaraki-ken 319-11, Japan

61. R. N. Hwang, Argonne National Laboratory, Reactor Analysis Division, BIdg 208, Argonne, IL 60439

62. R. P. Jacqmin, DER/SPRC/LEPH, Batiment 230, Centre d'Etudes de CADARACHE, 13108 Saint Paul-lez-Durance, France

63. N. Janeva, Bulgarian Academy of Sciences, 72, Boul, Tzarigradsko shosse, Sofia 1784, Bulgaria

64. L. Lois, 08 E23, U.S. Nuclear Regulatory Commission, 11555 Rockville Pike, Rockville, MD 20852-2746

65. G. Leinweber, Gaerttner LINAC Laboratory, Department of Environmental and Energy Engineering, Rensselaer Polytechnic Institute, Troy, NY 12180-3590

66. R. Little, X-CI, MS F663, Los Alamos National Laboratory, Los Alamos, NM 87545

67. C. Lubitz, Knolls Atomic Power Laboratory, P. O. Box 1072, Schenectady, NY 12301

68. R. E. MacFarlane,T-2, MS B243, Los Alamos National Laboratory, P. O. Box 1663, Los Alamos, NM 87545-1663

69. C. Mounier, CEN Saclay, DMT/SERMA/LENR, 91191 Gif Sur Yvette Cedex, France

70. M. C. Moxon, 3 Hyde Copse, Marcham, Abingdon, Oxfordshire, England

71. D. Muir, IAEA Nuclear Data Section, Wagramerstr. 5, P. O. Box 100, A-1400 Wien, Austria

72. C. W. Nilson, Office of Nuclear Regulatory Research, U.S. Nuclear Regulatory Commission, Mail Stop TWFN 9-F-33, Washington, DC 20555

73. C. Nordborg, OECD/NEA, Le Seine St-Germain 12, Boulevard Iles, 92130 Issy-les-Moulineaux, France

74. C. Raepsaet, CEN Saclay, DMT/SERMA/LEPP, 91191 Gif Sur Yvette Cedex, France

75. M. Salvatores, DRN/P, Batiment 707, C. E. CADARACHE, 13108 Saint Paul-lez-Durance, France

76. E. Sartori, OECD/NEA, Le Seine St-Germain 12, Boulevard Iles, 92130 Issy-les-Moulineaux, France

77. O. A. Shcherbakov, Petersburg Nuclear Physics Institute, 188350 Gatchina, Leningrad District, Russia

78. R. Shelley, Central Bureau for Nuclear Measurements, Steenweg op Retie, 2240 Geel, Belgium 
79. K. Shibata, Nuclear Data Center, Japan Atomic Energy Research Institute, Tokai-mura, Naka-gun, Ibaraki-ken 319-11, Japan

80. R. Slovacek, Gaerttner LINAC Laboratory, Department of Environmental and Energy Engineering, Rensselaer Polytechnic Institute, Troy, NY 12180-3590

81. A. B. Smith, Technology Development Division, D-207, AA115, Argonne National Laboratory 9700 South Cass Avenue Argonne, IL 60439

82. D. L. Smith, TD-207-DB116, Argonne National Laboratory, Argonne, IL 60544

83. H. Takano, Nuclear Data Center, Japan Atomic Energy Research Institute, Tokai-mura, Ibaraki-ken 319-11, Japan

84. J.J. Wagschal, Racah Institute of Physics, The Hebrew University of Jerusalem, 91904, Jerusalem, ISRAEL

85. H. Weigmann, Central Bureau for Nuclear Measurements, Steenweg op Retie, 2240 Geel, Belgium

86. C. Werner, Renssalaer Polytechnic Institute, Troy, NY 12180-3590

87. R. White, Lawrence Livermore National Laboratory, P. O. Box 808, Livermore, CA 94550

88. M. Williams, Nuclear Science Center, Louisiana State University, Baton Rouge, LA 70803 